\title{
The Soft X\#Ray Properties of a Complete Sample of Optically Selected Quasars. II. Final Results
}

\section{Citation}

Laor, Ari, Fabrizio Fiore, Martin Elvis, Belinda J. Wilkes, and Jonathan C. McDowell. 1997. "The Soft X\#Ray Properties of a Complete Sample of Optically Selected Quasars. II. Final Results." The Astrophysical Journal 477 (1) (March): 93-113. doi:10.1086/303696.

\section{Published Version}

doi:10.1086/303696

\section{Permanent link}

http://nrs.harvard.edu/urn-3:HUL.InstRepos:30248643

\section{Terms of Use}

This article was downloaded from Harvard University's DASH repository, and is made available under the terms and conditions applicable to Other Posted Material, as set forth at http:// nrs.harvard.edu/urn-3:HUL.InstRepos:dash.current.terms-of-use\#LAA

\section{Share Your Story}

The Harvard community has made this article openly available.

Please share how this access benefits you. Submit a story.

\section{Accessibility}


THE Astrophysical Journal, 477:93-113, 1997 March 1

(C) 1997. The American Astronomical Society. All rights reserved. Printed in U.S.A.

\title{
THE SOFT X-RAY PROPERTIES OF A COMPLETE SAMPLE OF OPTICALLY SELECTED QUASARS. II. FINAL RESULTS
}

ARI LAOR

Theoretical Astrophysics, Caltech 130-33, Pasadena, CA 91125; current address: Physics Department, Technion, Haifa 32000, Israel; laor@physics.technion.ac.il

FABRIZIO FIORE

Osservatorio Astronomico di Roma, via dell'Osservatorio 5, Monteporzio-Catone (RM) 00040, and SAX Scientific Data Center, via Corcolle 19, I-00131, Rome, Italy; fiore@susy.mporzio.astro.it

AND

Martin Elvis, Belinda J. Wilkes, and Jonathan C. McDowell

Harvard-Smithsonian Center for Astrophysics, 60 Garden Street, Cambridge, MA 02138; elvis@cfa.harvard.edu, belinda@cfa.harvard.edu, mcdowell@cfa.harvard.edu

Received 1996 June 6; accepted 1996 September 19

\begin{abstract}
We present the final results of a ROSAT PSPC program to study the soft X-ray emission properties of a complete sample of low- $z$ quasars. This sample includes all 23 quasars from the Bright Quasar Survey with $z \leq 0.400$ and $N_{\mathrm{HI}}^{\mathrm{Gal}}<1.9 \times 10^{20} \mathrm{~cm}^{-2}$. Pointed ROSAT PSPC observations were made for all quasars, yielding high signal-to-noise $(\mathrm{S} / \mathrm{N})$ spectra for most objects, which allowed an accurate determination of the spectral shape. The following main results were obtained:
\end{abstract}

1. The spectra of 22 of the 23 quasars are consistent, to within $\sim 30 \%$, with a single power-law model at rest-frame $0.2-2 \mathrm{keV}$. There is no evidence for significant soft excess emission with respect to the bestfit power law. We place a limit $\left(95 \%\right.$ confidence) of $\sim 5 \times 10^{19} \mathrm{~cm}^{-2}$ on the amount of excess foreground absorption by cold gas for most of our quasars. The limits are $\sim 1 \times 10^{19} \mathrm{~cm}^{-2}$ in the two highest $\mathrm{S} / \mathrm{N}$ spectra.

2. The mean $0.2-2 \mathrm{keV}$ continuum of quasars agrees remarkably well with an extrapolation of the mean 1050-350 A continuum recently determined by Zheng et al. (1996) for $z>0.33$ quasars. This suggests that there is no steep soft component below $0.2 \mathrm{keV}$.

3. Significant X-ray absorption $(\tau>0.3)$ by partially ionized gas ("warm absorber") in quasars is rather rare, occurring for $\$ 5 \%$ of the population, which is in sharp contrast to lower luminosity active galactic nuclei (AGNs), where significant absorption probably occurs for $\sim 50 \%$ of the population.

4. Extensive correlation analysis of the X-ray continuum emission parameters with optical emissionline parameters indicates that the strongest correlation is between the spectral slope $\alpha_{x}$ and the $\mathrm{H} \beta$ FWHM. A possible explanation for this remarkably strong correlation is a dependence of $\alpha_{x}$ on $L / L_{\mathrm{Edd}}$, as seen in Galactic black hole candidates.

5. The strong correlations between $\alpha_{x}$ and $L_{[\mathrm{O} \text { III] }}, \mathrm{Fe} \mathrm{II/H} \beta$, and the peak [O III] to $\mathrm{H} \beta$ flux ratio are verified. The physical origin of these correlations is still not understood.

6. There appears to be a distinct class of "X-ray-weak" quasars, which form $\sim 10 \%$ of the population (three out of 23), where the X-ray emission is smaller, by a factor of 10-30, than expected based on their luminosity at other bands and on their $\mathrm{H} \beta$ luminosity. These may be quasars in which the direct X-ray source is obscured and only scattered X-rays are observed.

7. Thin accretion disk models cannot reproduce the observed $0.2-2 \mathrm{keV}$ spectral shape, and they also cannot reproduce the tight correlation between the optical and soft X-ray emission. An as yet unknown physical mechanism must be maintaining a strong correlation between the optical and soft X-ray emission.

8. The $\mathrm{H} \mathrm{I} / \mathrm{He} \mathrm{I}$ ratio in the high Galactic latitude ISM must be within $20 \%$, and possibly within $5 \%$, of the total $\mathrm{H} / \mathrm{He}$ ratio of 10 , which indicates that $\mathrm{He}$ in the diffuse $\mathrm{H}$ II gas component of the interstellar medium is mostly ionized to He II or He III.

We finally note the intriguing possibility that although $\left\langle\alpha_{x}\right\rangle$ in radio-loud quasars $(-1.15 \pm 0.14)$ is significantly flatter than in radio-quiet quasars $(-1.72 \pm 0.09)$ the X-ray emission may not be related to the presence of radio emission. The difference in $\left\langle\alpha_{x}\right\rangle$ may result from the strong $\alpha_{x}$ versus H $\beta$ FWHM correlation and the tendency of radio-loud quasars to have broader $\mathrm{H} \beta$.

Subject headings: galaxies: active — galaxies: nuclei — quasars: general $-\mathrm{X}$-rays: galaxies

\section{INTRODUCTION}

Quasars emit most of their power in the ultraviolet to soft $\mathrm{X}$-ray regime. The position-sensitive proportional counter (PSPC) detector aboard ROSAT allowed a significantly improved study of the soft X-ray emission of quasars com- pared with earlier missions (some of which were not sensitive below $2 \mathrm{keV}$ ), such as HEAO-1, Einstein, EXOSAT, and Ginga (e.g., Mushotzky 1984; Wilkes \& Elvis 1987; Canizares \& White 1989; Comastri et al. 1992; Lawson et al. 1992; Williams et al. 1992; and a recent review by 
Mushotzky, Done, \& Pounds 1993). These earlier studies indicated that the X-ray emission above $1-2 \mathrm{keV}$ is well described by a power law with a spectral slope $\alpha_{x}=$ $d \ln f_{v} / d \ln v$ of about -0.5 for radio-loud quasars and about -1.0 for radio-quiet quasars. Large heterogeneous samples of AGNs were recently studied using the ROSAT PSPC by Walter \& Fink (1993) and by Wang, Brinkmann, \& Bergeron (1996). However, the objects studied in the papers mentioned above do not form a complete sample, and the available results may be biased by various selection effects that were not well defined a priori. In particular, most of the studied objects are nearby, intrinsically X-ray-bright AGNs.

To overcome the potential biases in existing studies, we initiated a ROSAT PSPC program to make an accurate determination of the soft X-ray properties of a well-defined and complete sample of quasars, selected independently of their X-ray properties. This program was designed to address the following questions:

1. What are the soft X-ray spectral properties of the lowredshift quasar population?

2. Are simple thin accretion disk models (e.g., Laor 1990) able to fit the observed optical/UV/soft X-ray continuum? Are other modifying mechanisms, such as a hot corona (e.g., Czerny \& Elvis 1987), required? Are models invoking optically thin free-free emission possible (e.g., Ferland, Korista, \& Peterson 1990; Barvainis 1993)?

3. Do the observed soft X-ray properties display any significant correlations with other properties of these quasars? Are these correlations compatible with various models for the continuum and line emission mechanisms?

Our sample includes all 23 quasars from the Bright Quasar Survey (BQS) sample (Schmidt \& Green 1983) with $z \leq 0.400$ and $N_{\mathrm{HI}}^{\mathrm{Gal}}<1.9 \times 10^{20} \mathrm{~cm}^{-2}$, where $N_{\mathrm{HI}}^{\mathrm{Gal}}$ is the $\mathrm{H}$ I Galactic column density as measured at $21 \mathrm{~cm}$. These selection criteria allow optimal study of soft X-ray emission at the lowest possible energy. The additional advantages of the BQS sample are that it has been extensively explored at other wavelengths (see Laor et al. 1994, hereafter Paper I, for further details) and that it includes only bright quasars, thus allowing high signal-to-noise $(\mathrm{S} / \mathrm{N}) \mathrm{X}$-ray spectra for most objects. The sample selection criteria are independent of the quasar's X-ray properties, and we thus expect our sample to be representative of the low-redshift, optically selected quasar population.

Preliminary results from the analysis of the first 10 quasars available to us were described in Paper I. Here we report the analysis of the complete sample, which allows us to address the three questions posed above. The outline of the paper is as follows. In $\S 2$ we describe the observations and the analysis of the spectra. Section 3 describes the analysis of correlations between the soft X-ray properties and other continuum and emission-line properties. In $\S 4$ we compare our results with other soft X-ray observations and discuss some of the implications. We conclude in $\S 5$ with answers to the questions raised above, and with some new questions to be addressed in future studies.

\section{OBSERVATIONS AND ANALYSIS OF THE SPECTRA}

The complete sample of 23 quasars is listed in Table 1 together with their redshifts, $m_{B}$ and $M_{B}$ magnitudes (calculated for $H_{0}=50 \mathrm{~km} \mathrm{~s}^{-1} \mathrm{Mpc}^{-1}, q=0.5$ ), $R$ (the radio-to-optical flux ratio), and $N_{\mathrm{HI}}^{\mathrm{Ga}}$. The redshifts and $m_{B}$ and $M_{B}$ magnitudes are taken from Schmidt \& Green (1983); $R$ is taken from Kellermann et al. (1989). Note that four of the 23 quasars in our sample are radio loud (defined here as $R \geq 10$ ).

TABLE 1

The Complete PG Quasar Soft X-Ray Survey SAmple ${ }^{\mathrm{a}}$

\begin{tabular}{|c|c|c|c|c|c|c|c|}
\hline Quasar & Other Name & $z^{\mathbf{b}}$ & $m_{B}{ }^{\mathrm{b}}$ & $M_{B}^{\mathrm{b}}$ & $R^{\mathrm{c}}$ & $\begin{array}{c}N_{\mathrm{HI}}^{\mathrm{Gal}} \\
\left(\times 10^{20} \mathrm{~cm}^{-2}\right)\end{array}$ & Reference $^{\mathrm{d}}$ \\
\hline PG $0947+396 \ldots \ldots$ & K347-45 & 0.206 & 16.40 & -24.06 & 0.25 & 1.92 & 1 \\
\hline PG $0953+414 \ldots \ldots$ & K $348-7$ & 0.239 & 15.05 & -25.73 & 0.44 & 1.12 & 2 \\
\hline PG $1001+054 \ldots \ldots$ & $\ldots$ & 0.161 & 16.13 & -23.79 & 0.50 & 1.88 & 3 \\
\hline PG $1048+342 \ldots \ldots$ & $\ldots$ & 0.167 & 15.81 & -24.19 & $\lesssim 0.10$ & 1.74 & 1 \\
\hline PG $1114+445 \ldots \ldots$ & $\ldots$ & 0.144 & 16.05 & -23.63 & 0.13 & 1.94 & 1 \\
\hline PG $1115+407 \ldots \ldots$ & $\ldots$ & 0.154 & 16.02 & -23.81 & 0.17 & 1.74 & 1 \\
\hline PG $1116+215 \ldots \ldots$ & TON 1388 & 0.177 & 15.17 & -24.96 & 0.72 & 1.44 & 3 \\
\hline PG $1202+281 \ldots \ldots$ & GQ COM & 0.165 & 15.02 & -24.96 & 0.19 & 1.72 & 3 \\
\hline PG $1216+069 \ldots \ldots$ & & 0.334 & 15.68 & -25.82 & 1.65 & 1.57 & 2 \\
\hline PG $1226+023 \ldots \ldots$ & $3 C 273$ & 0.158 & 12.86 & -27.02 & 1138 & 1.68 & 4 \\
\hline PG $1309+355 \ldots \ldots$ & TON 1565 & 0.184 & 15.45 & -24.76 & 18.0 & 1.01 & 1 \\
\hline PG $1322+659 \ldots \ldots$ & & 0.168 & 15.86 & -24.15 & 0.12 & 1.88 & 3 \\
\hline PG $1352+183 \ldots \ldots$ & PB 4142 & 0.158 & 15.71 & -24.17 & 0.11 & 1.84 & 3 \\
\hline PG $1402+261 \ldots \ldots$ & TON 182 & 0.164 & 15.57 & -24.39 & 0.23 & 1.42 & 3 \\
\hline PG $1411+442 \ldots \ldots$ & PB 1732 & 0.089 & 14.99 & -23.65 & 0.13 & 1.05 & 2 \\
\hline PG $1415+451 \ldots \ldots$ & & 0.114 & 15.74 & -23.43 & 0.17 & 0.96 & 1 \\
\hline PG $1425+267 \ldots \ldots$ & TON 202 & 0.366 & 15.67 & -26.03 & 53.6 & 1.54 & 3 \\
\hline PG $1427+480 \ldots \ldots$ & & 0.221 & 16.33 & -24.28 & 0.16 & 1.69 & 1 \\
\hline PG $1440+356 \ldots \ldots$ & Mrk 478 & 0.077 & 15.00 & -23.32 & 0.37 & 0.97 & 1 \\
\hline PG $1444+407 \ldots \ldots$ & & 0.267 & 15.95 & -25.07 & $\lesssim 0.08$ & 1.09 & 5 \\
\hline PG $1512+370 \ldots \ldots$ & 4C 37.43 & 0.371 & 15.97 & -25.76 & 190 & 1.40 & 4 \\
\hline PG $1543+489 \ldots \ldots$ & $\ldots$ & 0.400 & 16.05 & -25.84 & 0.15 & 1.68 & 2 \\
\hline PG $1626+554 \ldots \ldots$ & $\ldots$ & 0.133 & 16.17 & -23.34 & 0.11 & 1.55 & 3 \\
\hline
\end{tabular}

${ }^{\text {a }}$ All PG sample quasars (i.e., $M_{B}<-23$ ) with $z \leq 0.400$ and $N_{\mathrm{HI}}^{\mathrm{Gal}}<1.9 \times 10^{20}$.

${ }^{\mathrm{b}}$ From Schmidt \& Green 1983; $M_{B}$ calculated assuming $H_{0}=50 \mathrm{~km} \mathrm{~s}^{-1} \mathrm{Mpc}^{-1}$ and $q_{0}=0.5$.

c Radio $(6 \mathrm{~cm})$ to optical (4400 ̊) flux ratio, taken from Kellermann et al. 1989. Radio-loud quasars have $R \gtrsim 10$.

${ }^{\mathrm{d}} N_{\mathrm{HI}}^{\text {Gal }}$ taken from (1) Murphy et al. 1996; (2) Lockman \& Savage 1995; (3) Elvis, Lockman, \& Wilkes 1989;

(4) Savage et al. 1993; (5) F. J. Lockman 1995, private communication. 
The Galaxy becomes optically thick below $0.2 \mathrm{keV}$ for the typical high Galactic column of $3 \times 10^{20} \mathrm{~cm}^{-2}$ (Dickey \& Lockman 1990; Morrison \& McCammon 1983), and accurate values of $N_{\mathrm{HI}}^{\mathrm{Gal}}$ are therefore crucial even for our low$N_{\mathrm{HI}}^{\mathrm{Gal}}$ sample. The $N_{\mathrm{HI}}^{\mathrm{Gal}}$ values given in column (7) of Table 1 are taken from Elvis, Lockman, \& Wilkes (1989), Savage et al. (1993), Lockman \& Savage (1995), and the recent extensive measurements by Murphy et al. (1996). All these measurements of $N_{\mathrm{HI}}^{\mathrm{Gal}}$ were made with the 140 foot $(43 \mathrm{~m})$ telescope of the NRAO at Green Bank, using the "bootstrapping" stray radiation correction method described by Lockman, Jahoda, \& McCammon (1986), which provides an angular resolution of $21^{\prime}$ and an uncertainty of $\Delta N_{\mathrm{HI}}^{\mathrm{Gal}}=1 \times 10^{19} \mathrm{~cm}^{-2}$ (and possibly lower for our low $N_{\mathrm{H}}$ quasars). This uncertainty introduces a flux error of $10 \%$ at $0.2 \mathrm{keV}, 30 \%$ at $0.15 \mathrm{keV}$, and nearly a factor of 2 at $0.1 \mathrm{keV}$. Thus, reasonably accurate fluxes can be obtained down to $\sim 0.15 \mathrm{keV}$. Note that Murphy et al. (1996) include accurate $N_{\mathrm{HI}}^{\mathrm{Gal}}$ measurements for about 220 AGNs, including most AGNs observed by ROSAT, which would be very useful for eliminating the significant systematic uncertainty in the PSPC spectral slope that must be present when a low-accuracy $N_{\mathrm{HI}}^{\mathrm{Gal}}$ is used.

Table 2 lists the PSPC observations of all the quasars. For the sake of completeness we include also the 10 quasars already reported in Paper I. All sources were detected, and their net source counts range from 93 to 38,015, with a median value of about 1900 counts. The PROS software package was used to extract the source counts. Table 2 includes the exposure times, the dates of the observations, the net number of counts and their statistical error, the count rate, the radius of the circular aperture used to extract the source counts, the offset of the X-ray position from the center of the PSPC field of view, the ROSAT sequence identification number, and the SASS version used for the calibration of the data. All objects, except one, are typically within $15^{\prime \prime}$ of the center of the PSPC field of view, so all the identifications are secure. The one exception is PG $1440+356$, where the pointing was offset by $40^{\prime}$ from the position of the quasar (Gondhalekar et al. 1994). Note that the exposure times are uncertain by about $4 \%$ as the result of a number of possible systematic errors, as described by Fiore et al. (1994). The typically large number of counts for each object allows an accurate determination of the spectral slope for most objects, as described below.

Model fits to the extracted number of source counts per pulse-invariant (PI) channel, $N_{\mathrm{ch}}^{\mathrm{ob}}$, were carried out using the XSPEC software package. PI channels 1-12 of the original 256 channel spectra $(E<0.11 \mathrm{keV})$, were ignored, since they are not well calibrated and are inherently uncertain because of the large Galactic optical depth. The 1993 January PSPC calibration matrix was used for observations made after 1991 October 14, and earlier observations were fitted with the 1992 March calibration matrix. The best-fit model parameters are obtained by $\chi^{2}$ minimization. Nearby channels were merged when $N_{\mathrm{ch}}^{\mathrm{ob}}<10$. A $1 \%$ error was added in quadrature to the statistical error in $N_{\mathrm{ch}}^{\mathrm{ob}}$, to take into account possible systematic calibration errors (see Paper I for more complete details).

\subsection{A Single Power Law}

As in Paper I, we fitted each spectrum with a single power law of the form $f_{E}=e^{-N_{\mathrm{H}} \sigma_{E}} f_{0} E^{\alpha_{x}}$, where $f_{E}$ is the flux density, $\sigma_{E}$ is the absorption cross section per $\mathrm{H}$ atom (Morrison \& McCammon 1983), $f_{0}$ is the flux density at $1 \mathrm{keV}$, and $E$ is in units of $\mathrm{keV}$. We make three different fits for each object, with (1) $N_{\mathrm{H}}$ a free parameter, (2) $N_{\mathrm{H}}=N_{\mathrm{HI}}^{\mathrm{Gal}}$, (3) $N_{\mathrm{H}}=N_{\mathrm{HI}}^{\mathrm{Gal}}$, and $0.47 \leq E \leq 2.5 \mathrm{keV}$, i.e., using only the hard ROSAT band (channels 12-34 of the rebinned 34 channel spectra). A comparison of fits 1 and 2 allows us to determine whether

TABLE 2

JOURNAL OF ROSAT PSPC OBSERVATIONS

\begin{tabular}{|c|c|c|c|c|c|c|c|c|c|}
\hline $\begin{array}{l}\text { Quasar } \\
\text { (PG) }\end{array}$ & $z$ & $\begin{array}{c}\text { Exposure }^{a} \\
\text { (s) }\end{array}$ & $\begin{array}{c}\text { Dates } \\
\text { (Start-End) }\end{array}$ & Counts $^{b}$ & Counts $s^{-1 b}$ & $\begin{array}{c}r^{\mathrm{c}} \\
(\operatorname{arcmin})\end{array}$ & $\begin{array}{l}\text { Offset }^{\mathrm{d}} \\
(\operatorname{arcsec})\end{array}$ & $\begin{array}{l}\text { Sequence } \\
\text { Number }\end{array}$ & $\begin{array}{c}\text { SASS } \\
\text { Version }\end{array}$ \\
\hline $0947+396 \ldots \ldots$ & 0.206 & 14283 & 1993 Oct $26-27$ & $5345 \pm 78$ & 0.374 & 3.0 & 9.9 & 70136700 & 6.8 \\
\hline $0953+414 \ldots \ldots$ & 0.239 & 7070 & 1992 Apr 30 & $4770 \pm 74$ & 0.675 & 3.0 & 26.0 & 700526 & 5.7 \\
\hline $1001+054 \ldots \ldots$ & 0.161 & 8868 & 1992 May 17-Jun 2 & $93.4 \pm 16$ & 0.011 & 2.0 & 10.8 & 700774 & 5.7 \\
\hline $1048+342 \ldots \ldots$ & 0.167 & 8072 & 1993 Nov 6 & $1624 \pm 44$ & 0.201 & 2.5 & 6.9 & 70136800 & 6.8 \\
\hline $1114+445 \ldots \ldots$ & 0.144 & 7373 & 1993 Jun 6-7 & $777 \pm 30$ & 0.105 & 2.0 & 15.0 & 700800 & 6.4 \\
\hline $1115+407 \ldots \ldots$ & 0.154 & 6495 & 1993 May 20 & $1934 \pm 156$ & 0.298 & 2.5 & 14.8 & 700801 & 6.4 \\
\hline $1116+215 \ldots \ldots$ & 0.177 & 24942 & 1991 May $29-30$ & $24272 \pm 162$ & 0.973 & 3.0 & 20.0 & 700228 & 5.4 \\
\hline $1202+281 \ldots \ldots$ & 0.165 & 19777 & 1991 Jun 4-5 & $7882 \pm 95$ & 0.399 & 2.5 & 18.3 & 700232 & 5.3 \\
\hline $1216+069 \ldots \ldots$ & 0.334 & 3464 & 1991 Dec $20-21$ & $1370 \pm 39$ & 0.395 & 3.0 & 17.9 & 700021 & 5.6 \\
\hline $1322+659 \ldots \ldots$ & 0.168 & 8389 & 1992 Nov $30-$ Dec 1 & $4187 \pm 67$ & 0.499 & 2.5 & 5.4 & 700803 & 6.2 \\
\hline $1352+183 \ldots \ldots$ & 0.158 & 5521 & 1992 Jul 22 & $2285 \pm 51$ & 0.414 & 3.0 & 13.1 & 700804 & 5.8 \\
\hline $1402+261 \ldots \ldots$ & 0.164 & 4357 & 1992 Jan 7-Jul 18 & $4153 \pm 67$ & 0.953 & 3.0 & 7.8 & 700226 & 5.11 \\
\hline $1411+442 \ldots \ldots$ & 0.089 & 25319 & 1991 Jun $26-27$ & $1031 \pm 40$ & 0.041 & 2.0 & 12.6 & 700248 & 5.3 \\
\hline $1415+451 \ldots \ldots$ & 0.114 & 7432 & 1992 Jul 16 & $2514 \pm 54$ & 0.338 & 3.0 & 11.6 & 700805 & 5.8 \\
\hline $1427+480 \ldots \ldots$ & 0.221 & 6780 & 1993 Jul 15-16 & $1104 \pm 35$ & 0.163 & 2.5 & 12.0 & 70137000 & 6.4 \\
\hline $1440+356 \ldots \ldots$ & 0.077 & 2393 & 1992 Jan 17 & $4934 \pm 74$ & 2.062 & 7.0 & 2430 & 700559 & 5.4 \\
\hline $1444+407 \ldots \ldots$ & 0.267 & 5620 & 1993 Jul 19-20 & $1881 \pm 46$ & 0.335 & 2.5 & 12.0 & 70137100 & 6.4 \\
\hline $1543+489 \ldots \ldots$ & 0.400 & 12759 & 1992 Aug 21-23 & $1375 \pm 313$ & 0.108 & 2.5 & 22.8 & $700808 / 9$ & 5.8 \\
\hline $1626+554 \ldots \ldots$ & 0.133 & 2312 & 1993 Aug 3-4 & $2294 \pm 48$ & 0.992 & 2.0 & 18.4 & 70137200 & 6.4 \\
\hline $1226+023 \ldots \ldots$ & 0.158 & 6243 & 1991 Dec $14-15$ & $38015 \pm 197$ & 6.089 & 3.0 & 8.0 & 700191 & 5.4 \\
\hline $1309+355 \ldots \ldots$ & 0.184 & 2528 & 1992 Jul 6 & $593 \pm 25$ & 0.235 & 2.0 & 16.0 & 700802 & 5.7 \\
\hline $1425+267 \ldots \ldots$ & 0.366 & 6716 & 1993 Jul 18-21 & $224 \pm 18$ & 0.033 & 1.5 & 18.0 & 70136900 & 6.4 \\
\hline $1512+370 \ldots \ldots$ & 0.371 & 5230 & 1992 Aug $12-17$ & $1498 \pm 40$ & 0.286 & 2.0 & 13.0 & 700807 & 5.8 \\
\hline
\end{tabular}

${ }^{a}$ Uncertain by about $4 \%$ due to systematic errors.

${ }^{b}$ Net source counts in PI channels 11-245.

c Effective aperture for integration of counts.

${ }^{\mathrm{d}}$ Offset of X-ray position from the center of the PSPC field of view. 
there is evidence for a significant intrinsic absorption excess or emission excess relative to a single power-law fit with $N_{\mathrm{H}}=N_{\mathrm{HI}}^{\mathrm{Gal}}$. This comparison also allows us, as is further shown in $\S 4.2$, to determine whether the $21 \mathrm{~cm}$ measurement of $N_{\mathrm{HI}}^{\mathrm{Gal}}$ is a reliable measure of the Galactic soft X-ray opacity. A comparison of fits 2 and 3 allows us to look for a dependence of the power-law slope on energy.
Table 3 provides the results of fits 1-3 described above. The table includes the 13 objects not reported in Paper I, and six objects from Paper I for which we only now have the accurate $N_{\mathrm{HI}}^{\mathrm{Gal}}$ values. For each fit we give the best-fitting spectral slope $\alpha_{x}$, the normalization of the power-law flux at $1 \mathrm{keV}\left(f_{0}\right)$, the best-fitting $N_{\mathrm{H}}$, the $\chi^{2}$ of the fit $\left(\chi_{\mathrm{fit}}^{2}\right)$, the number of degrees of freedom (dof), and the probability for

TABLE 3

SINGLE POWER-LAW MODELS $\left(f_{E}=e^{-N_{\mathrm{H} \sigma_{E}}} f_{0} E^{\alpha_{x}}\right)$

\begin{tabular}{|c|c|c|c|c|c|c|}
\hline $\begin{array}{l}\text { Name } \\
\text { (PG) }\end{array}$ & $\mathrm{Fit}^{\mathrm{a}}$ & $\alpha_{x}^{b}$ & $\begin{array}{c}f_{0}{ }^{c} \\
\left(\times 10^{-4}\right)\end{array}$ & $\begin{array}{c}N_{\mathrm{H}}^{\mathrm{d}} \\
\left(10^{20} \mathrm{~cm}^{-2}\right)\end{array}$ & $\chi_{\mathrm{fit}}^{2} / \mathrm{dof}$ & $\operatorname{Pr}\left(\chi^{2} \geq \chi_{\mathrm{fit}}^{2}\right)$ \\
\hline \multirow[t]{3}{*}{$0947+396 \ldots \ldots$} & 1 & $-1.36_{-0.09}^{+0.10}$ & $7.64 \pm 0.27$ & $1.54 \pm 0.22$ & $26.51 / 26$ & 0.43 \\
\hline & 2 & $-1.51 \pm 0.02$ & $7.64 \pm 0.20$ & $1 . \overline{9} 2$ & $32.10 / 27$ & 0.23 \\
\hline & 3 & $-1.44 \pm 0.07$ & $7.68 \pm 0.18$ & 1.92 & $21.27 / 18$ & 0.27 \\
\hline \multirow{3}{*}{$0953+414 \ldots \ldots$} & 1 & $-1.71 \pm 0.12$ & $9.30 \pm 0.50$ & $1.50 \pm 0.25$ & $14.31 / 26$ & 0.97 \\
\hline & 2 & $-1.57 \pm 0.03$ & $9.33 \pm 0.14$ & 1.12 & $18.12 / 27$ & 0.90 \\
\hline & 3 & $-1.62 \pm 0.11$ & $9.25 \pm 0.32$ & 1.12 & $9.56 / 18$ & 0.95 \\
\hline $1001+054 \ldots \ldots$ & 1 & $-2.80_{-0.58}^{+1.07}$ & $0.048_{-0.039}^{+0.06}$ & 1.88 & $2.02 / 4$ & 0.73 \\
\hline \multirow[t]{3}{*}{$1048+342 \ldots \ldots$} & 1 & $-1.27 \pm 0.21$ & $4.23 \pm 0.32$ & $1.47 \pm 0.45$ & $14.86 / 23$ & 0.90 \\
\hline & 2 & $-1.39 \pm 0.05$ & $4.24 \pm 0.21$ & $1 . \overline{7} 4$ & $15.68 / 24$ & 0.90 \\
\hline & 3 & $-1.33 \pm 0.15$ & $4.24 \pm 0.20$ & 1.74 & $10.84 / 14$ & 0.70 \\
\hline \multirow[t]{3}{*}{$1114+445 \ldots \ldots$} & 1 & $-1.13_{-0.31}^{\overline{+0}} \cdot .24$ & $1.97 \pm 0.20$ & $1.04 \pm 0.62$ & $59.72 / 25$ & $1.1 \times 10^{-4}$ \\
\hline & 2 & $-1.55 \pm 0.06$ & $1.91 \pm 0.13$ & 1.94 & $63.85 / 26$ & $5 \times 10^{-5}$ \\
\hline & 3 & $-0.17 \pm 0.31$ & $1.96 \pm 0.13$ & 1.94 & $36.16 / 17$ & $4.4 \times 10^{-3}$ \\
\hline \multirow[t]{3}{*}{$1115+407 \ldots \ldots$} & 1 & $-1.77 \pm 0.17$ & $3.82 \pm 0.28$ & $1.48 \pm 0.36$ & $24.54 / 21$ & 0.27 \\
\hline & 2 & $-1.89 \pm 0.04$ & $3.79 \pm 0.19$ & $1 . \overline{74}$ & $25.73 / 22$ & 0.26 \\
\hline & 3 & $-1.86 \pm 0.14$ & $3.81 \pm 0.20$ & 1.74 & $11.28 / 13$ & 0.59 \\
\hline \multirow[t]{3}{*}{$1116+215 \ldots \ldots$} & 1 & $-1.73 \pm 0.05$ & $13.1 \pm 0.28$ & $1.43 \pm 0.10$ & $38.13 / 29$ & 0.12 \\
\hline & 2 & $-1.73 \pm 0.01$ & $13.1 \pm 0.16$ & $1 . \overline{4} 4$ & $38.16 / 30$ & 0.15 \\
\hline & 3 & $-1.69 \pm 0.04$ & $13.2 \pm 0.15$ & 1.44 & $24.04 / 21$ & 0.29 \\
\hline \multirow[t]{3}{*}{$1202+281 \ldots \ldots$} & 1 & $-1.23 \pm 0.08$ & $9.93 \pm 0.27$ & $1.88 \pm 0.20$ & $20.79 / 28$ & 0.83 \\
\hline & 2 & $-1.22 \pm 0.02$ & $9.87 \pm 0.17$ & 1.72 & $22.45 / 29$ & 0.80 \\
\hline & 3 & $-1.22 \pm 0.05$ & $9.88 \pm 0.17$ & 1.72 & $18.83 / 20$ & 0.53 \\
\hline \multirow{3}{*}{$1216+069 \ldots \ldots$} & 1 & $-1.52 \pm 0.20$ & $8.01 \pm 0.57$ & $1.96_{-0.43}^{+0.48}$ & $24.14 / 25$ & 0.51 \\
\hline & 2 & $-1.36 \pm 0.04$ & $7.93 \pm 0.38$ & 1.57 & $26.01 / 26$ & 0.46 \\
\hline & 3 & $-1.43 \pm 0.13$ & $7.93 \pm 0.41$ & 1.57 & $17.05 / 17$ & 0.45 \\
\hline \multirow{3}{*}{$1309+335 \ldots \ldots$} & 1 & $-1.725 \pm 0.31$ & $3.00 \pm 0.40$ & $1.43_{-0.50}^{+0.62}$ & $20.99 / 22$ & 0.52 \\
\hline & 2 & $-1.51 \pm 0.07$ & $3.02 \pm 0.26$ & 1.01 & $22.63 / 23$ & 0.48 \\
\hline & 3 & $-1.42+0.25$ & $3.03 \pm 0.40$ & 1.01 & $10.92 / 14$ & 0.69 \\
\hline \multirow[t]{3}{*}{$1402+261 \ldots \ldots$} & 1 & $-1.79 \pm 0.13$ & $10.00 \pm 0.67$ & $1.16 \pm 0.22$ & $26.02 / 25$ & 0.41 \\
\hline & 2 & $-1.93+0.03$ & $9.86+0.04$ & 1.42 & $29.13 / 26$ & 0.31 \\
\hline & 3 & $-1.57 \pm 0.14$ & $10.3 \pm 0.4$ & 1.42 & $10.16 / 16$ & 0.86 \\
\hline \multirow{3}{*}{$1411+442 \ldots \ldots$} & 1 & $-2.14_{-0.29}^{+0.27}$ & $0.31 \pm 0.05$ & $1.42 \pm 0.54$ & $25.64 / 20$ & 0.18 \\
\hline & 2 & $-1.97 \pm 0.07$ & $0.31 \pm 0.03$ & 1.05 & $26.46 / 21$ & 0.19 \\
\hline & 3 & $-1.87 \pm 0.27$ & $0.30 \pm 0.03$ & 1.05 & $14.53 / 12$ & 0.27 \\
\hline \multirow[t]{3}{*}{$1415+451 \ldots \ldots$} & 1 & $-1.59 \pm 0.13$ & $3.32 \pm 0.25$ & $0.67_{-0.19}^{+0.22}$ & $19.98 / 24$ & 0.70 \\
\hline & 2 & $-1.74 \pm 0.04$ & $3.28 \pm 0.18$ & 0.96 & $22.91 / 25$ & 0.58 \\
\hline & 3 & $-1.57 \pm 0.14$ & $3.40 \pm 0.17$ & 0.96 & $15.31 / 16$ & 0.50 \\
\hline \multirow[t]{3}{*}{$1425+267 \ldots \ldots$} & 1 & $-0.71_{-0.58}^{+0.85}$ & $0.84 \pm 0.18$ & $1.0_{-1.0}^{+2.0}$ & $13.2 / 9$ & 0.15 \\
\hline & 2 & $-0.94+0.16$ & $0.85+0.11$ & 1.54 & $13.47 / 10$ & 0.20 \\
\hline & 3 & $-0.13 \pm 0.64$ & $0.81 \pm 0.13$ & 1.54 & $5.50 / 4$ & 0.24 \\
\hline \multirow[t]{3}{*}{$1427+480 \ldots \ldots$} & 1 & $-1.37 \pm 0.27$ & $3.25 \pm 0.31$ & $1.60 \pm 0.60$ & $19.82 / 23$ & 0.65 \\
\hline & 2 & $-1.41 \pm 0.05$ & $3.26 \pm 0.22$ & 1.69 & $19.98 / 24$ & 0.70 \\
\hline & 3 & $-1.24 \pm 0.20$ & $3.28 \pm 0.20$ & 1.69 & $13.79 / 15$ & 0.54 \\
\hline \multirow{3}{*}{$1440+356 \ldots \ldots$} & 1 & $-2.38 \pm 0.13$ & $19.2 \pm 1.5$ & $1.49_{-0.20}^{+0.22}$ & $20.53 / 24$ & 0.66 \\
\hline & 2 & $-2.08 \pm 0.03$ & $20.2 \pm 0.9$ & 0.97 & $37.60 / 25$ & 0.05 \\
\hline & 3 & $-2.17 \pm 0.13$ & $19.3 \pm 1.0$ & 0.97 & $11.21 / 16$ & 0.80 \\
\hline \multirow[t]{3}{*}{$1444+407 \ldots \ldots$} & 1 & $-1.86 \pm 0.20$ & $2.91 \pm 0.34$ & $1.00_{-0.29}^{+0.35}$ & $14.46 / 19$ & 0.76 \\
\hline & 2 & $-1.91+0.05$ & $2.89+0.22$ & 1.09 & $14.66 / 20$ & 0.80 \\
\hline & 3 & $-2.14 \pm 0.19$ & $2.82 \pm 0.23$ & 1.09 & $4.39 / 11$ & 0.96 \\
\hline \multirow[t]{3}{*}{$1543+489 \ldots \ldots$} & 1 & $-2.57_{-0.31}^{+0.27}$ & $0.98 \pm 0.13$ & $2.67_{-0.56}^{+0.67}$ & $29.93 / 23$ & 0.15 \\
\hline & 2 & $-2.11 \pm 0.05$ & $1.02 \pm 0.08$ & 1.68 & $38.62 / 24$ & 0.03 \\
\hline & 3 & $-2.19 \pm 0.20$ & $0.99 \pm 0.10$ & 1.68 & $20.95 / 15$ & 0.14 \\
\hline \multirow{3}{*}{$1626+554 \ldots \ldots$} & 1 & $-1.68 \pm 0.14$ & $12.9 \pm 0.9$ & $1.26 \pm 0.29$ & $17.78 / 23$ & 0.77 \\
\hline & 2 & $-1.94 \pm 0.04$ & $12.8 \pm 0.6$ & 1.82 & $25.26 / 24$ & 0.39 \\
\hline & 3 & $-1.83 \pm 0.15$ & $13.1 \pm 0.6$ & 1.82 & $16.49 / 15$ & 0.35 \\
\hline
\end{tabular}

a The best-fit single power-law model for (1) PI channels 11-245, and $N_{\mathrm{H}}$ a free parameter; (2) PI channels 11-245, and $N_{\mathrm{H}}=N_{\mathrm{HI}}^{\mathrm{Gal}}$; (3) PI channels 47-245, and $N_{\mathrm{H}}=N_{\mathrm{HI}}^{\mathrm{Gal}}$.

b The error is $1 \sigma$ for either two interesting parameters (fit 1 ) or one interesting parameter (fits 2 and 3 ).

${ }^{c}$ In units of $\mathrm{keV} \mathrm{cm}^{-2} \mathrm{~s}^{-1} \mathrm{keV}^{-1}$, or, equivalently, $6.626 \times 10^{-27} \mathrm{ergs} \mathrm{cm}^{-2} \mathrm{~s}^{-1} \mathrm{~Hz}^{-1}$, at $1 \mathrm{keV}$.

${ }^{\mathrm{d}}$ In fit 1 the $N_{\mathrm{H}}$ error is $1 \sigma$ for two interesting parameters. In fits 2 and 3 the $N_{\mathrm{H}}$ error is assumed to be zero. 
$\chi^{2} \geq \chi_{\mathrm{fit}}^{2}$. The errors $\Delta \alpha_{x}$ and $\Delta N_{\mathrm{H}}$ in fit 1 were calculated by making a grid search for models with $\Delta \chi^{2}=2.30$, as appropriate for a $1 \sigma$ confidence level for two interesting parameters (e.g., Press et al. 1989). The error on the slope, $\Delta \alpha_{x}$, in fits 2 and 3 is calculated by requiring $\Delta \chi^{2}=1.0$ (i.e., $68 \%$ for one interesting parameter). We neglect the effect of $\Delta N_{\mathrm{HI}}^{\mathrm{Gal}}$ on $\Delta \alpha_{x}$ in fits 2 and 3 , since we use accurate values of $N_{\mathrm{HI}}^{\mathrm{Gal}}$ for all objects.

The observed and best-fit spectra for the 13 quasars not reported in Paper I are displayed in Figure 1. There are three panels for each object. The upper panel displays the observed count rate per $\mathrm{keV}$ as a function of channel energy; the histogram represents the expected rate from the best-fit power-law model, $N_{\mathrm{ch}}^{\mathrm{ob}}$, with $N_{\mathrm{H}}$ a free parameter (fit 1). The middle panel displays $\Delta / \sigma$, where $\Delta=N_{\mathrm{ch}}^{\mathrm{ob}}-N_{\mathrm{ch}}^{\text {mod }}$ and $\sigma$ is the standard error in $N_{\mathrm{ch}}^{\mathrm{ob}}$. This plot helps indicate what features in the spectrum are significant. The lower
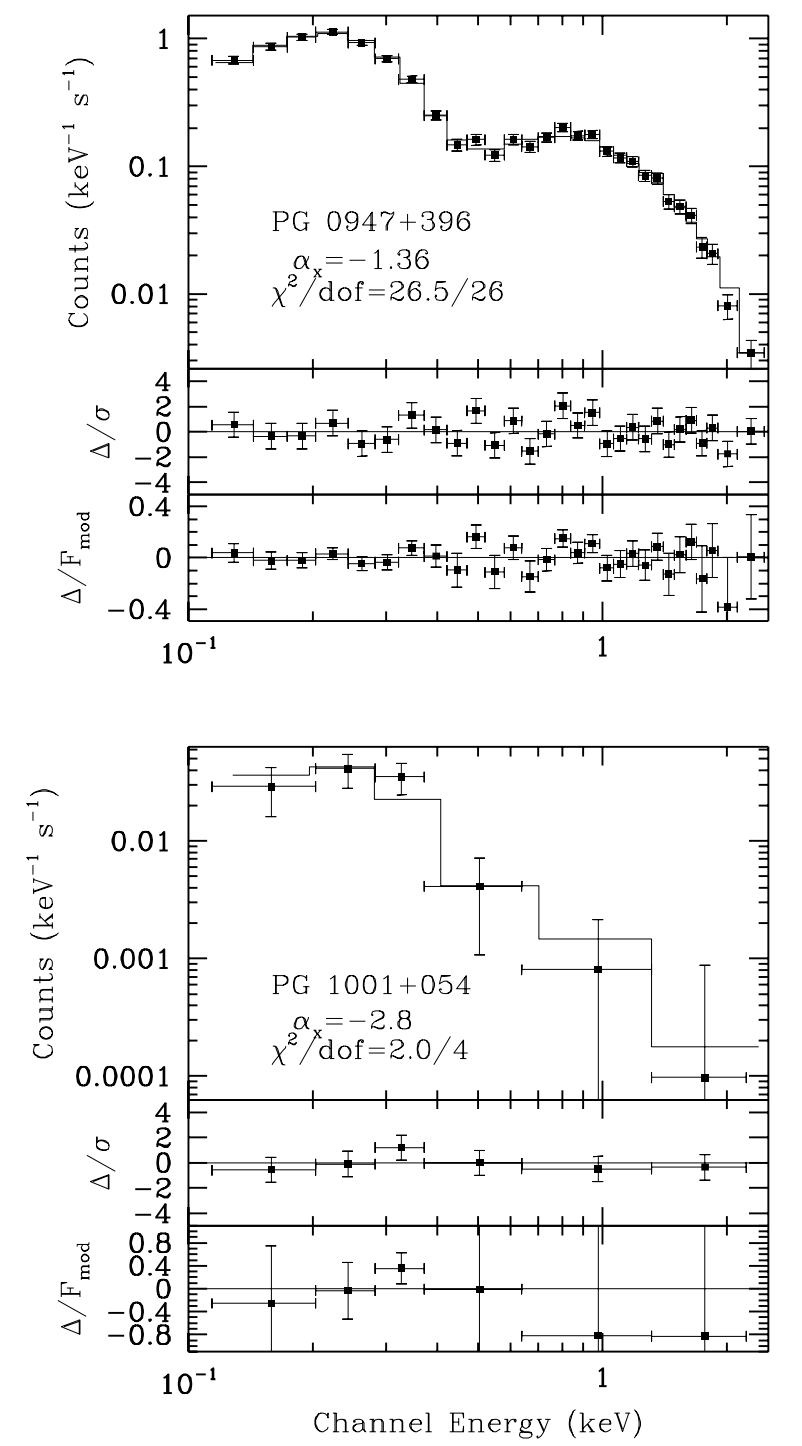

panel displays the fractional deviations from the expected flux or, equivalently, $\Delta / N_{\mathrm{ch}}^{\mathrm{mod}}$, which indicates the fractional amplitude of the observed features.

As shown in Table 3, in all 13 objects the simple powerlaw model with $N_{\mathrm{HI}}^{\mathrm{Gal}}$ (fit 2) provides an acceptable fit [i.e., $\mathrm{Pr}$ $\left.\left(\chi^{2} \geq \chi_{\mathrm{fit}}^{2}\right)>0.01\right]$. Note in particular the spectrum of PG $1116+215$, which despite the very high $\mathrm{S} / \mathrm{N}$ available (24,272 net counts) shows no deviations from a simple power law above a level of $\lesssim 10 \%$. In Paper I a simple power-law model could not provide an acceptable fit to three of the 10 quasars, although in two of them the apparent features could not be fitted with a simple physical model, and in one of them this may be due to calibration errors (see $\S 4.1$ ).

As mentioned above, a comparison of the free $N_{\mathrm{H}}$ fit (fit 1) with the $N_{\mathrm{HI}}^{\mathrm{Gal}}$ fit (fit 2) allows us to look for evidence for an absorption or an emission excess. We measure the statistical
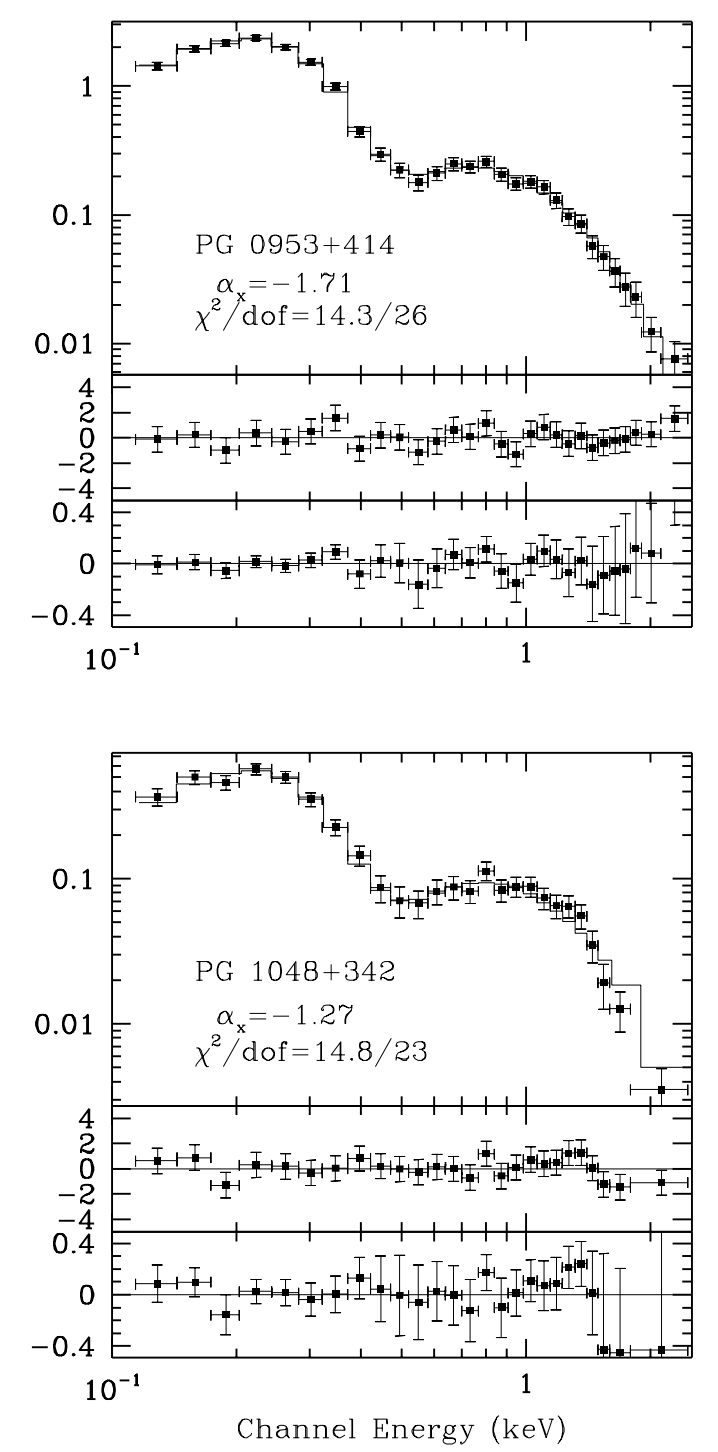

FIG. $1 a$

Fig. 1.-Observed vs. single power-law fit PSPC spectra of the 13 quasars not reported in Paper I. The upper panel for each object shows the observed count rate (points with error bars), and the expected count rate using the best-fit single power-law model with a free $N_{\mathrm{H}}$ (solid-line histogram). The middle panel shows the observed minus the expected flux in units of standard deviations, and the lower panel for each object shows the fractional deviations from the expected flux. The best-fit $\alpha_{x}$, the fit $\chi^{2}$, and the number of degrees of freedom (dof) are indicated for each object. The spectra of all quasars are consistent with a simple power law. Note in particular the high $\mathrm{S} / \mathrm{N}$ spectrum of PG $1116+215$, where intrinsic features below $1 \mathrm{keV}$ must have an amplitude of less than $\simeq 10 \%$. 

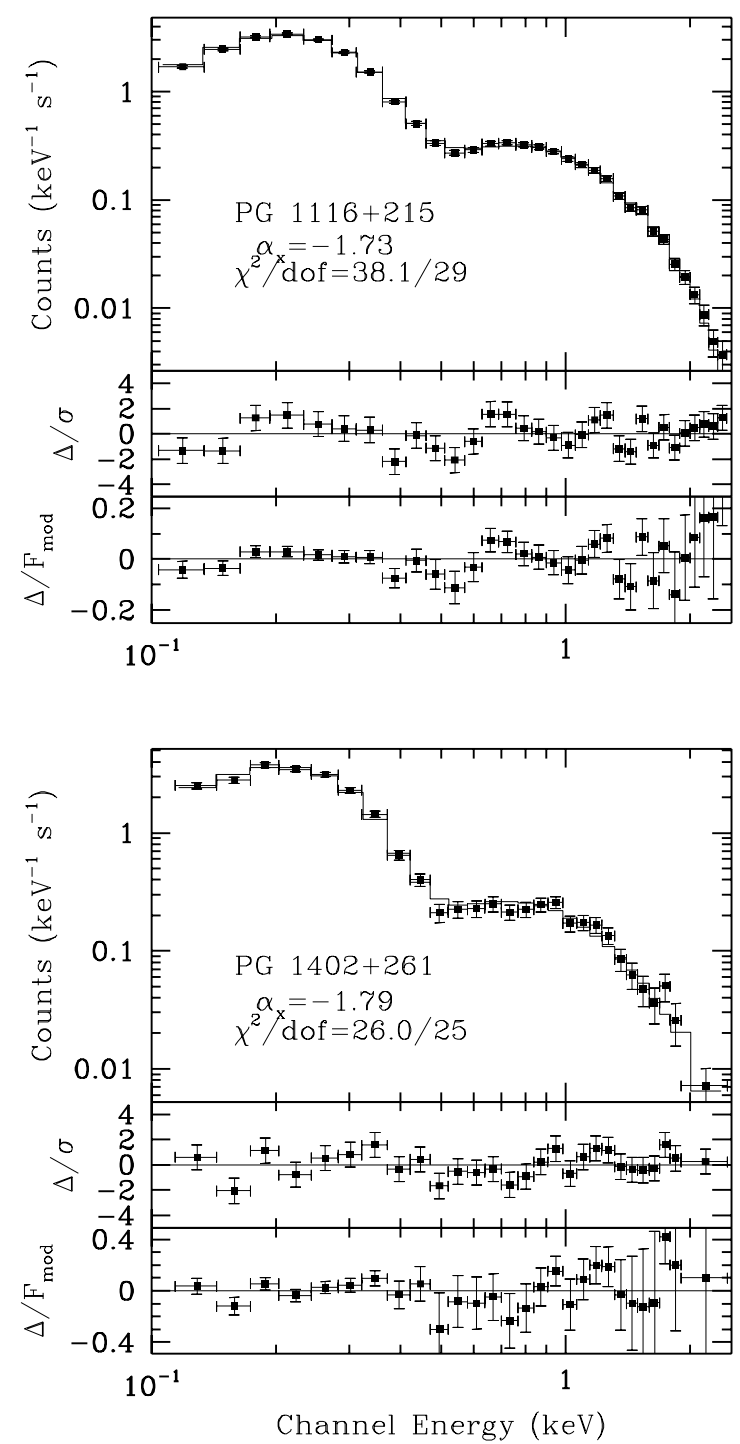
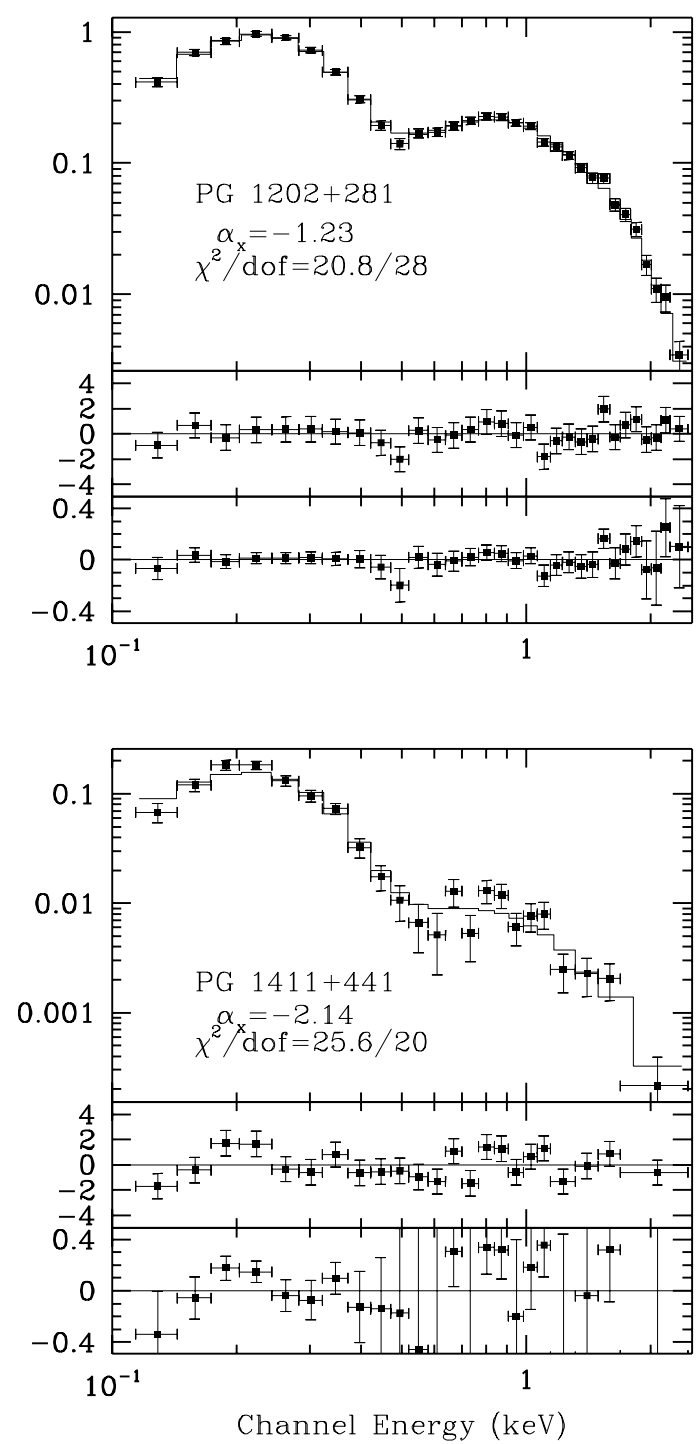

FIG. $1 b$

significance of the reduction in $\chi_{\mathrm{fit}}^{2}$ with the addition of $N_{\mathrm{H}}$ as a free parameter using the $F$-test (Bevington 1969). In PG $1440+356$ we find a significant reduction with $\mathrm{Pr}=$ $7.5 \times 10^{-4}(F=12.98$ for $26 \mathrm{dof})$, where $\operatorname{Pr}$ is the probability that the reduction in $\chi^{2}$ is not statistically significant (calculated using the FTEST routine in Press et al. 1989). The $N_{\mathrm{H}}$ obtained in fit 1 suggests intrinsic absorption of about $5 \times 10^{19} \mathrm{~cm}^{-2}$ above the Galactic absorption. However, unlike all other objects, PG $1440+356$ was observed significantly off axis (see Table 2), and some small systematic calibration errors may be present there. Note also that the $\chi_{\mathrm{fit}}^{2}$ of the fit with $N_{\mathrm{HI}}^{\mathrm{Gal}}(\mathrm{Pr}=0.05)$ is still acceptable. We therefore cannot conclude that an extra absorber must be present in PG $1440+356$. Marshall et al. (1996) found a very steep slope $(\alpha=-4.7 \pm 0.65)$ in PG $1440+35$ at $0.1-0.15 \mathrm{keV}(80-120 \AA)$ using the Extreme Ultraviolet Explorer. There is no indication for such a component in the PSPC spectrum below $0.15 \mathrm{keV}$ (Fig. 1c). However, given the very low sensitivity of the PSPC below $0.15 \mathrm{keV}$, such a steep soft component may still be consistent with the PSPC spectrum. In the other 12 objects the free $N_{\mathrm{H}}$ fit does not provide a significant improvement (i.e.,
$\operatorname{Pr}>0.01$ ), and thus there is no clear evidence for either intrinsic absorption or low-energy excess emission above a simple power law.

Figure 2 compares the Galactic $N_{\mathrm{H}}$ deduced from the accurate $21 \mathrm{~cm}$ measurements with the best-fit X-ray column deduced using the free $N_{\mathrm{H}}$ fit. The straight line represents equal columns. The $\chi^{2}$ of the $N_{\mathrm{H}}(21 \mathrm{~cm})=$ $N_{\mathrm{H}}$ (X-ray) model is 31.9 for 22 dof (PG 1001+054 was not included because of the low $\mathrm{S} / \mathrm{N}$ ), which is acceptable at the $8 \%$ level. This result demonstrates that there is no significant excess absorption over the Galactic value in any of our objects. It is interesting to note that in our highest $\mathrm{S} / \mathrm{N}$ spectra, those of PG $1116+215$ and PG $1226+023$, $N_{\mathrm{H}}$ (X-ray) is determined to a level of $(0.8-1) \times 10^{19} \mathrm{~cm}^{-2}$ and is still consistent with $N_{\mathrm{H}}(21 \mathrm{~cm})$, indicating that the two methods agree to better than $10 \%$.

The average hard ROSAT band $(0.5-2 \mathrm{keV})$ slope for the complete sample is $-1.59 \pm 0.08$ (excluding PG $1114+445$, which is affected by a warm absorber, and PG $1001+054$ and PG $1425+267$, for which the $\mathrm{S} / \mathrm{N}$ is very low). This slope is not significantly different from the average slope for the full ROSAT band, $-1.63 \pm 0.07$. 

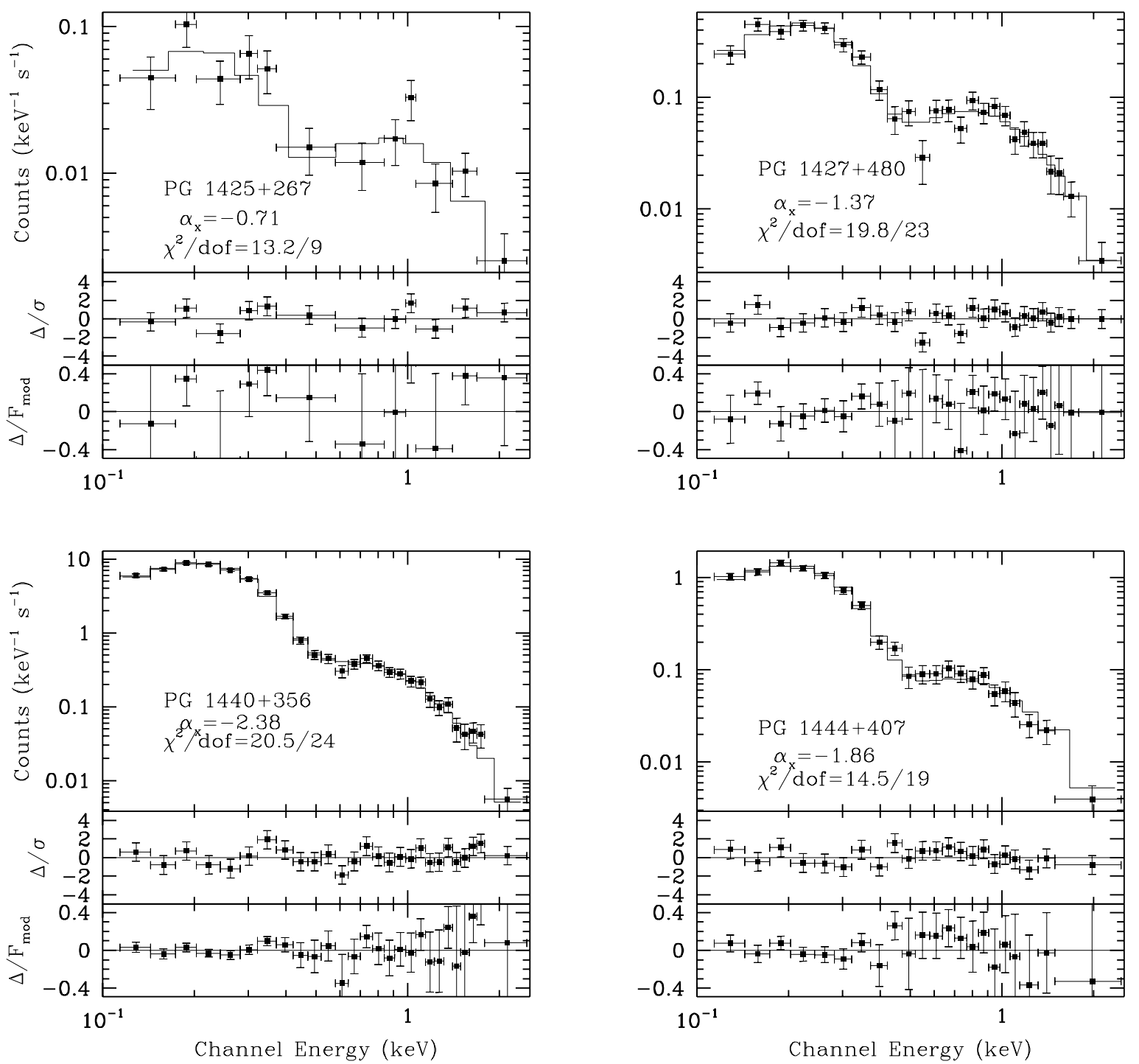

FIG. 1c

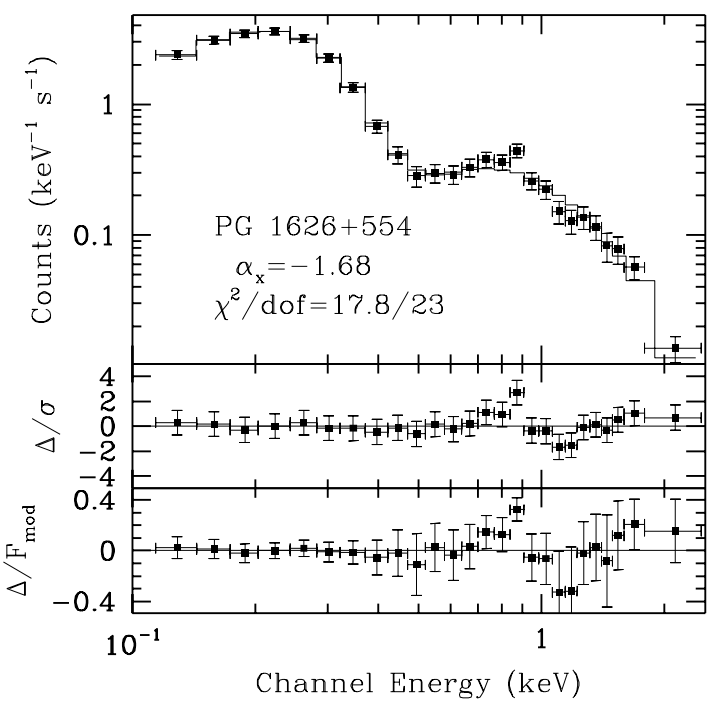

FIG. $1 d$ 


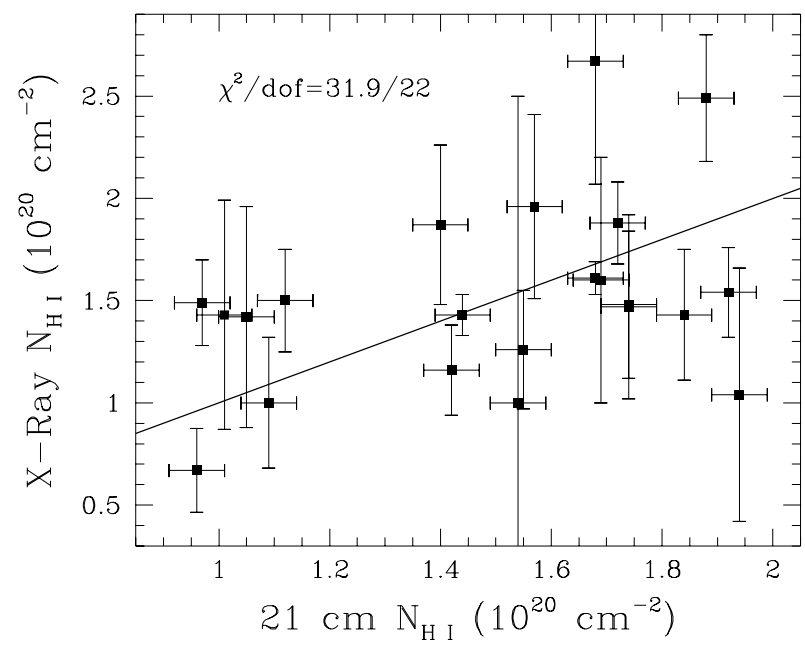

FIG. 2.-The H I column determined by $21 \mathrm{~cm}$ measurements vs. the best-fit $\mathrm{H}$ I column determined by a power-law fit to the quasars' PSPC spectrum. The assumption that both values are equal, indicated by the straight line, is acceptable at the $8 \%$ level. Note, in particular, the two highest $\mathrm{S} / \mathrm{N}$ objects, which deviate from the straight line by less than $1 \times 10^{19} \mathrm{~cm}^{-2}$. The agreement between the two measures of $N_{\mathrm{H}}$ indicates a lack of intrinsic cold gas absorption in quasars, and that $\mathrm{H} \mathrm{I} / \mathrm{He} \mathrm{I} \simeq \mathrm{H} / \mathrm{He}$ in the ISM at high Galactic latitudes.

Spectral fits to the PSPC data of some of the objects in our complete sample have already been reported by Gondhalekar et al. (1994), Ulrich \& Molendi (1996), Rachen, Mannheim, \& Biermann (1996), and Wang et al. (1996). The results of the single power-law fit with a free $N_{\mathrm{H}}$ in these papers are all consistent with our results for the overlapping objects. The only discrepancy is with PG $1444+407$ for which, although both the present authors and Wang et al. (1996) find a similar slope, Wang et al. find evidence for absorption while we find no such evidence. For a simple power-law fit to PG 1444+407 Wang et al. (1996) find $\chi^{2}=26$ for 20 dof, which is acceptable only at the $17 \%$ level ( $\sim 1.4 \sigma$ level), while we find for such a fit $\chi^{2}=14.7$ for 20 dof, which is acceptable at the $80 \%$ level.

As discussed in Paper I (§ 5.1.3), the difference in spectral slopes at hard (2-10 keV) and soft X-rays raises the possibility that $\alpha_{x}$ may be changing within the PSPC band itself. The individual spectra are well fitted by a simple power law, and thus any spectral curvature must be consistent with zero. Stronger constraints on the spectral curvature may be obtained by measuring the average curvature parameter $(\beta$, defined in Paper I) for the complete sample, since the random error in the mean is smaller by $N^{1 / 2} \sim 5$ than the random error for individual objects. Unfortunately, the PSPC calibration uncertainty at low energy, discussed in Paper I, introduces a systematic error in $\beta$ (which obviously does not cancel out as $N^{1 / 2}$ ) and, as shown in Paper I, does not allow a reliable determination of the curvature parameter. We therefore did not try to constrain the spectral curvature parameter in this paper.

\section{CORRELATION ANALYSIS}

Table 4 presents eight of the 12 rest-frame continuum parameters and seven of the 18 emission-line parameters used for the correlation analysis. The spectral slopes are defined [the flux subscript indicates $\log v(\mathrm{~Hz})]$ as $\alpha_{o}=$ $\log \left(f_{15} / f_{14.5}\right) / 0.5(1-0.3 \mu \mathrm{m}), \alpha_{o x}=\log \left(f_{17.685} / f_{15}\right) / 2.685$ $(3000 \mathrm{~A}-2 \mathrm{keV})$, and $\alpha_{o s}=\log \left(f_{16.861} / f_{15}\right) / 1.861(3000$
$\AA-0.3 \mathrm{keV}$ ). The X-ray continuum parameters are from fit 2 and from Paper I. The near-IR and optical continuum parameters are taken from Neugebauer et al. (1987). The emission-line parameters were taken from Boroson \& Green (1992). Luminosities were calculated assuming $H_{0}=$ $50 \mathrm{~km} \mathrm{~s}^{-1} \mathrm{Mpc}^{-1}$ and $q_{0}=0.5$.

The four additional continuum parameters that are omitted from Table 5 for the sake of brevity are $\alpha_{i r x}=$ $\log \left(f_{16.861} / f_{14.25}\right) / 2.611 \quad(1.69 \mu \mathrm{m}-2 \quad \mathrm{keV}), \quad \alpha_{i r s}=\log$ $\left(f_{17.685} / f_{14.25}\right) / 3.435(1.69 \mu \mathrm{m}-0.3 \mathrm{keV})$, radio luminosity (Kellermann et al. 1989), and $1 \mu \mathrm{m}$ luminosity (Neugebauer et al. 1987). The 11 emission-line parameters not detailed in Table 5 are [O III] EW, Fe II EW, H $\beta$ EW, He II EW, $[\mathrm{O} \mathrm{III}] / \mathrm{H} \beta$ and $\mathrm{He}$ II/ $/ \mathrm{H} \beta$ flux ratios, [O III] peak flux to $\mathrm{H} \beta$ peak flux ratio, radio to optical flux ratio, and the $\mathrm{H} \beta$ asymmetry, shape, and shift parameters. All these 11 parameters are listed in Table 2 of Boroson \& Green (1992).

The significance of the correlations was tested using the Spearman rank-order correlation coefficient $\left(r_{\mathrm{s}}\right)$, which is sensitive to any monotonic relation between the two variables. A summary of the main correlation coefficients and their two-sided significance is given in Table 5.

\subsection{Significance Level}

In Paper I the correlation analysis was carried out using 10 objects, and only relatively strong correlations $\left(r_{\mathrm{s}} \geq\right.$ $0.76)$ could be detected at the required significance level $(\operatorname{Pr} \leq 0.01)$. Here, with 23 objects, $\operatorname{Pr} \leq 0.01$ corresponds to $r_{\mathrm{S}} \geq 0.52$, and we can thus test for the presence of weaker correlations and check whether the correlations suggested in Paper I remain significant. We have searched for correlations among the 12 continuum emission parameters, and between these 12 parameters and the 18 emission-line parameters listed above, which gives a total of 294 different correlations. One thus expects about 1 spurious correlation with $\operatorname{Pr} \leq 3.4 \times 10^{-3}$ in our analysis, and for a significance level of $1 \%$ one would now have to go to $\operatorname{Pr} \leq 3.4 \times 10^{-5}$ rather than $\operatorname{Pr} \leq 1 \times 10^{-2}$. However, we find that there are actually 42 correlations with $\operatorname{Pr} \leq 3.4 \times 10^{-3}$, rather than just one, in our sample. Thus, the probability that any one of them is the spurious one is only $2.4 \%$, and the significance level of these correlations is reduced by a factor of 7 $(=0.024 / 0.0034)$ rather than a factor of 300 . Below we assume that correlations with $\operatorname{Pr} \leq 1 \times 10^{-3}$ are significant at the $1 \%$ level (there are 30 correlations with $\operatorname{Pr} \leq 1 \times 10^{-3}$, versus an expected number of 0.3 ). Thus, given the large number of correlations we looked at, we can only test reliably for correlations with $r_{\mathrm{s}} \geq 0.64$ (which corresponds to $\operatorname{Pr} \leq 1 \times 10^{-3}$ for 23 data points).

\subsection{The Near-IR to $X$-Ray Energy Distribution}

A comparison of the rest-frame spectral energy distributions of all 23 quasars is shown in Figure 3. The 3-0.3 $\mu \mathrm{m}$ continuum is from Neugebauer et al. (1987), and the $0.2-2$ $\mathrm{keV}$ continuum is from Paper I and from this paper. The upper panel shows the absolute luminosities, and the two lower panels the luminosity normalized to unity at $\log v=14.25$ for radio-quiet quasars and for radio-loud quasars. Note the relatively small dispersion in the normalized $0.3 \mathrm{keV}(\log v=16.861)$ luminosity. The outlying objects are labeled. PG $1626+554$ is the only object where a steep $\alpha_{x}$ is clearly associated with a strong soft excess (relative to the near-IR flux). In other objects a steep $\alpha_{x}$ tends to be associated with a low $2 \mathrm{keV}$ flux. This trend is 


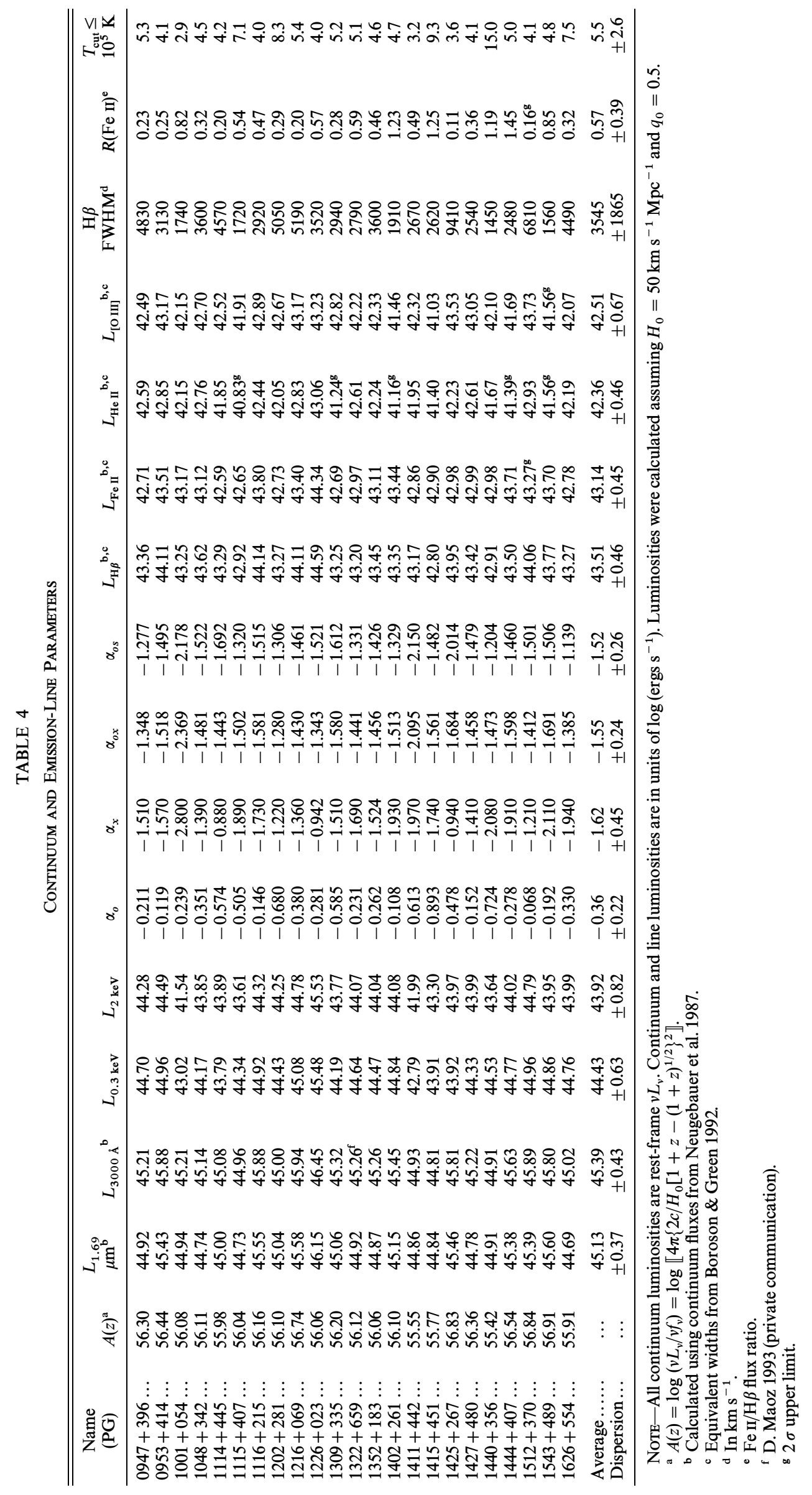




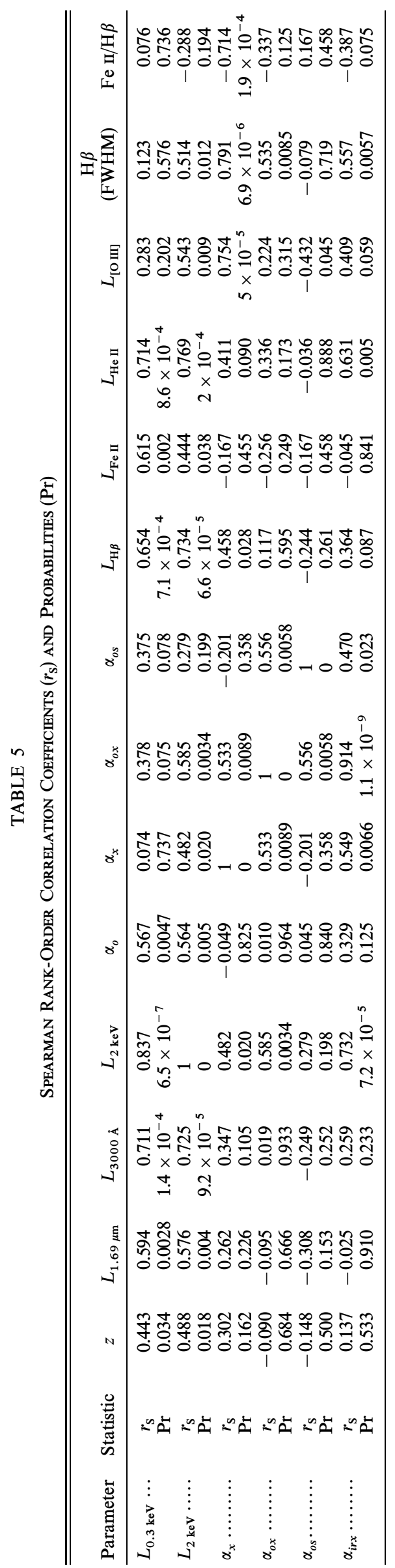




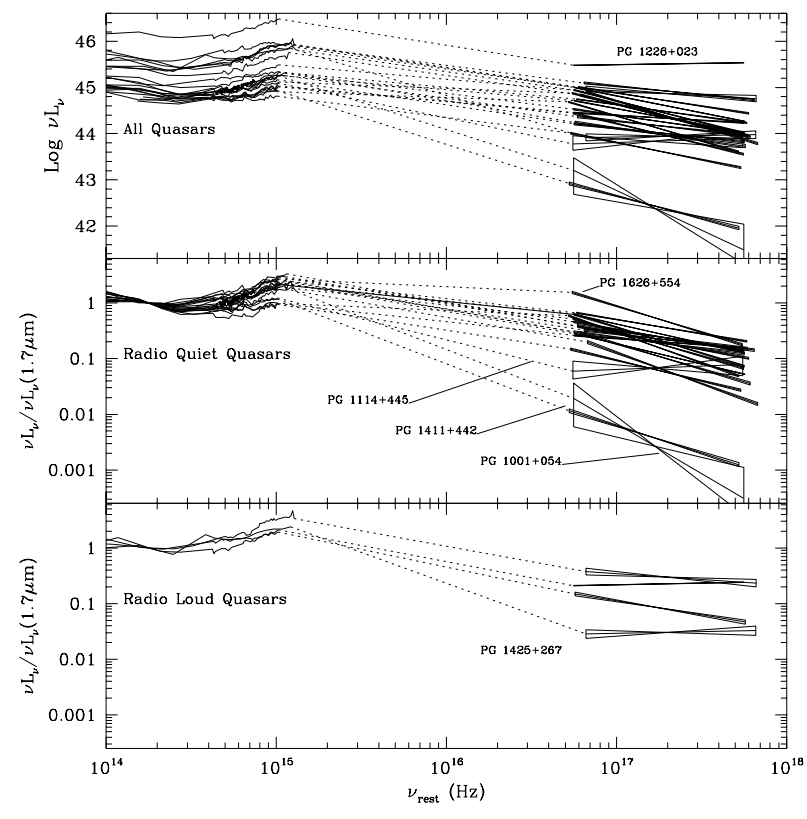

Fig. 3.-Comparison of the spectral energy distribution of all 23 quasars. Upper panel: Absolute luminosity. Middle panel: Luminosity of the 19 RQQs normalized to unity at $\log v=14.25$ (the longest wavelength available for all objects). Note that PG $1626+554$ is the only quasar for which a steep $\alpha_{x}$ is associated with a strong soft excess; in all other objects a steep $\alpha_{x}$ is associated with a low $2 \mathrm{keV}$ flux. Note also the two lowest spectra (PG 1001+054 and PG 1411+442), which appear to form a distinct group of "X-ray-weak" quasars. Lower panel: As in middle panel, for the four RLQs. PG 1425+267 may also be an "X-ray-weak" quasar.

also suggested by the presence of a marginally significant correlation between $\alpha_{x}$ and $\alpha_{o x}\left(r_{\mathrm{S}}=0.533, \operatorname{Pr}=0.0089\right.$; see below), and the absence of a significant correlation between $\alpha_{x}$ and $\alpha_{o s}\left(r_{\mathrm{S}}=-0.201, \operatorname{Pr}=0.36\right)$.

The X-ray luminosity distribution appears to be bimodal, with two quasars, PG $1001+054$ and PG $1411+442$, being a factor of 30 weaker than the mean radio-quiet quasar. These two quasars appear to form a distinct group of " $\mathrm{X}$ ray-weak quasars." The statistics for the radio-loud quasars (RLQs) are much poorer, and there is no well-defined mean, but PG $1425+267$ may be a similar X-ray-weak RLQ.

\subsection{Correlations with Emission-Line Properties}

Figure 4 presents the correlations between the hard X-ray luminosity, $L_{2 \mathrm{kev}}(\log v=17.685)$ or the soft X-ray luminosity, $L_{0.3 \mathrm{keV}}$, and the luminosity of $\mathrm{H} \beta$, [O III], He II, or $\mathrm{Fe}$ II. The value of $r_{\mathrm{S}}$ and the two-sided significance level (Pr) of $r_{\mathrm{S}}$ are indicated above each panel. Upper limits were not included in the correlations. Thus, the actual correlations for $\mathrm{He}$ II, where there are five upper limits, are likely to be smaller than found here (there is only one upper limit for [O III] and $\mathrm{Fe}$ II, and none for $\mathrm{H} \beta$ ). Excluding $\mathrm{He}$ II, the $\mathrm{X}$-ray luminosity is most strongly correlated with $L_{\mathrm{H} \beta}\left(r_{\mathrm{S}}=\right.$ $0.734, \mathrm{Pr}=6.6 \times 10^{-5}$ ). We note in passing that $L_{\mathrm{H} \beta}$ has an even stronger correlation with the luminosity at $3000 \AA$ $\left(r_{\mathrm{S}}=0.866, \operatorname{Pr}=9 \times 10^{-8}\right)$ and with the near-IR luminosity at $1 \mu \mathrm{m}\left(r_{\mathrm{s}}=0.810, \operatorname{Pr}=2.7 \times 10^{-6}\right)$.

The position of the X-ray-weak quasars is marked in Figure 4. Both PG 1001+054 and PG 1411+442 appear to have an X-ray luminosity weaker by a factor of about 30 compared to other quasars with similar $L_{\mathrm{H} \beta}$. PG $1425+267$ is also weaker by a factor of $\sim 10$ compared with the other
RLQs. These ratios are the same as those found above in $\S 3.2$, based on the spectral energy distribution.

Figures $5 a-5 d$ display various emission parameters that correlate with $\alpha_{x}$ (as obtained with $N_{\mathrm{H}}=N_{\mathrm{HI}}^{\mathrm{Gal}}$ ). The FWHM of $\mathrm{H} \beta, L_{[\mathrm{O} \text { III] }}$, the $\mathrm{Fe} \mathrm{II} / \mathrm{H} \beta$ flux ratio, and the ratio of $[\mathrm{O} \mathrm{III}]$ peak flux to $\mathrm{H} \beta$ peak flux (as defined by Boroson $\&$ Green) are the emission-line parameters that correlate most strongly with $\alpha_{x}$. As found in Paper I, all the $\alpha_{x}$ versus emission-line correlations become significantly weaker when we use $\alpha_{x}$ obtained with the free $N_{\mathrm{H}}$ fit.

The X-ray-weak quasars are labeled in the $\alpha_{o x}$ versus $\alpha_{x}$ correlation in Figure 5e. As expected, they have a steeper than expected $\alpha_{o x}$ for their $\alpha_{x}$. The last parameter shown in Figure $5 f$ is $1.5 L_{14.25}^{1 / 2} \Delta v^{-2}$, where $\Delta v=\mathrm{H} \beta$ FWHM. This parameter is related, under some assumptions, to the luminosity in Eddington units, as further discussed in $\S 4.7$.

\section{DISCUSSION}

\subsection{The Soft $X$-Ray Spectral Shape}

We find an average spectral index $\left\langle\alpha_{x}\right\rangle=-1.62 \pm 0.09$ for the complete sample of 23 quasars, where the error here and below is the uncertainty in the mean. This slope is consistent with the mean slope $\left\langle\alpha_{x}\right\rangle=-1.57 \pm 0.06$ that we found for the subsample of 24 quasars out of the 58 AGNs analyzed by Walter \& Fink (1993; the other 34 AGNs in their sample are Seyfert galaxies as defined by Véron-Cetty \& Véron 1991). A similar average slope of $-1.65 \pm 0.07$ was found by Schartel et al. (1996) for 72 quasars from the LBQS sample detected in the ROSAT all sky survey (RASS). Puchnarewicz et al. (1996) find a significantly flatter mean slope, $\left\langle\alpha_{x}\right\rangle=-1.07 \pm 0.06$, in a large sample of 108 soft X-ray-selected (0.5-2 keV) AGNs. Part of the difference is related to the exclusion of counts below $0.5 \mathrm{keV}$, which selects against steep $\alpha_{x}$ quasars, but this bias cannot explain the much flatter $\alpha_{o x}$ in their sample. As discussed by Puchnarewicz et al., their sample appears to include a large proportion of highly reddened quasars (see further discussion in $\S 4.2$ ).

RLQs are known to have a flatter $\alpha_{x}$ than radio-quiet quasars (RQQs) at energies above the PSPC band (e.g., Wilkes \& Elvis 1987; Lawson et al. 1992). We find $\left\langle\alpha_{x}\right\rangle=$ $-1.72 \pm 0.09$ for the 19 RQQs, and $\left\langle\alpha_{x}\right\rangle=-1.15 \pm 0.14$ for the four RLQs in our sample. We find a similar trend using the Walter $\&$ Fink (1993) quasar data, where $\left\langle\alpha_{x}\right\rangle=$ $-1.61 \pm 0.08$ for the RQQs and $\left\langle\alpha_{x}\right\rangle=-1.36 \pm 0.08$ for the RLQs. A similar difference between RQQs and RLQs was found by Ciliegi \& Maccacaro (1996) in PSPC spectra of a sample of 63 AGNs extracted from the Einstein extended medium-sensitivity survey sample. We therefore conclude that the trend observed at harder X-rays also extends down to the $0.2-2 \mathrm{keV}$ band. Schartel et al. (1996) stacked PSPC images of 147 RQQs and 32 RLQs, finding for the sum images $\left\langle\alpha_{x}\right\rangle=-1.65 \pm 0.18$ for the RQQs and $\left\langle\alpha_{x}\right\rangle=-1.00 \pm 0.28$ for the RLQs. However, the mean redshift of their objects is $\sim 1.3$, and thus their results apply to the $\sim 0.45-4.5 \mathrm{keV}$ band.

As discussed in Paper I, the ROSAT PSPC indicates a significantly different soft X-ray spectral shape for quasars compared with earlier results obtained by the Einstein imaging proportional counter (IPC) and EXOSAT lowenergy and medium-energy (LE + ME) detectors (e.g., Wilkes \& Elvis 1987; Masnou et al. 1992; Comastri et al. 1992; Saxton et al. 1993; Turner \& Pounds 1989; Kruper, 

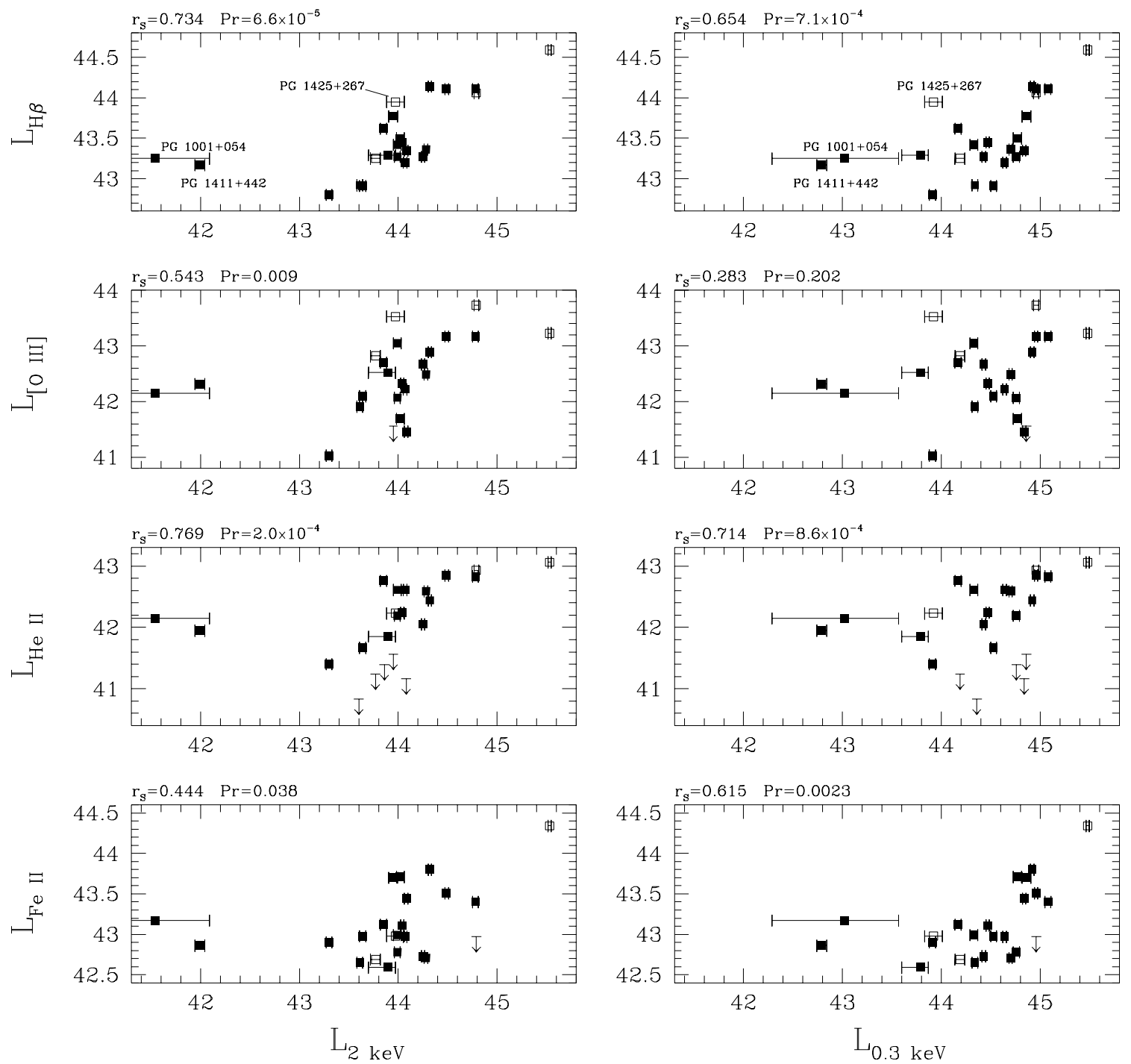

FIG. 4.-Correlations of line luminosity vs. X-ray luminosity. Open symbols are for radio-loud quasars. The Spearman rank-order correlation coefficient $\left(r_{\mathrm{S}}\right)$ and the two sided significance level $\left(P_{r}\right)$ are indicated above each panel. The strongest correlation is displayed by the $\mathrm{H} \beta$ luminosity (which correlates even better with the optical and near IR luminosity). Note the position of the X-ray-weak quasars. These appear to be fainter by factors of $10-30$ in the X-ray than expected based on their $L_{\mathrm{H} \beta}$. This factor is similar to the deficiency seen in Fig. 3.

Urry, \& Canizares 1990). In particular, earlier missions suggested that the hard X-ray slope (Lawson et al. 1992; Williams et al. 1992) extends down to $\sim 0.5 \mathrm{keV}$, with a steep rise at lower energy. Here we find that the $0.2-2 \mathrm{keV}$ spectrum is fitted well by a single power law with Galactic absorption. This indicates that (1) the break between the soft and hard X-ray slope must occur well above $0.5 \mathrm{keV}$, (2) the break must be gradual, and (3) there is no steep soft component with significant flux down to $\sim 0.2 \mathrm{keV}$. ASCA observations of two of the quasars in our sample, 3C 273 by Yaqoob et al. (1994) and PG $1116+215$ by Nandra et al. (1996), find, as expected, significantly flatter spectra above 2 $\mathrm{keV}$. However, the exact break energy cannot be accurately determined from the $A S C A$ spectra because of likely calibration uncertainties below $1 \mathrm{keV}$.

As mentioned in Paper I, the different Einstein IPC and EXOSAT LE + ME results may be traced back to the combined effect of the lower sensitivity of these instruments below $\sim 0.5 \mathrm{keV}$, and possibly some calibration errors. Small systematic errors in the PSPC response function appear to be present below $0.2 \mathrm{keV}$ (Fiore et al. 1994), and this instrument is thought to be significantly better calibrated at low energy than earlier instruments.

No significant spectral features are present in the PSPC spectra of any of 13 additional quasars reported here, indicating that intrinsic features must have an amplitude of less than $10 \%-20 \%$. Note in particular the high $\mathrm{S} / \mathrm{N}$ spectrum of PG $1116+215$, where the number of counts is about 12 times the median sample counts, yet this spectrum is still consistent with a simple power law. For the complete sample we find that only one quasar, PG $1114+445$, has a significant physical feature that is well described by a warm absorber model. In two other quasars, PG $1226+023$ and PG $1512+370$, there are significant features below $0.5 \mathrm{keV}$ (Paper I). In the case of PG $1512+370$ the features are at a level of $\sim 30 \%$, and in PG $1226+023$ they are at a level of $\lesssim 10 \%$ and may well be due to small calibration errors. This result is consistent with the result of Fiore et al.(1994), who found that a simple power law provides an acceptable fit to the individual spectra of six high S/N PSPC quasar spectra.

A composite optical to hard X-ray spectral energy dis- 

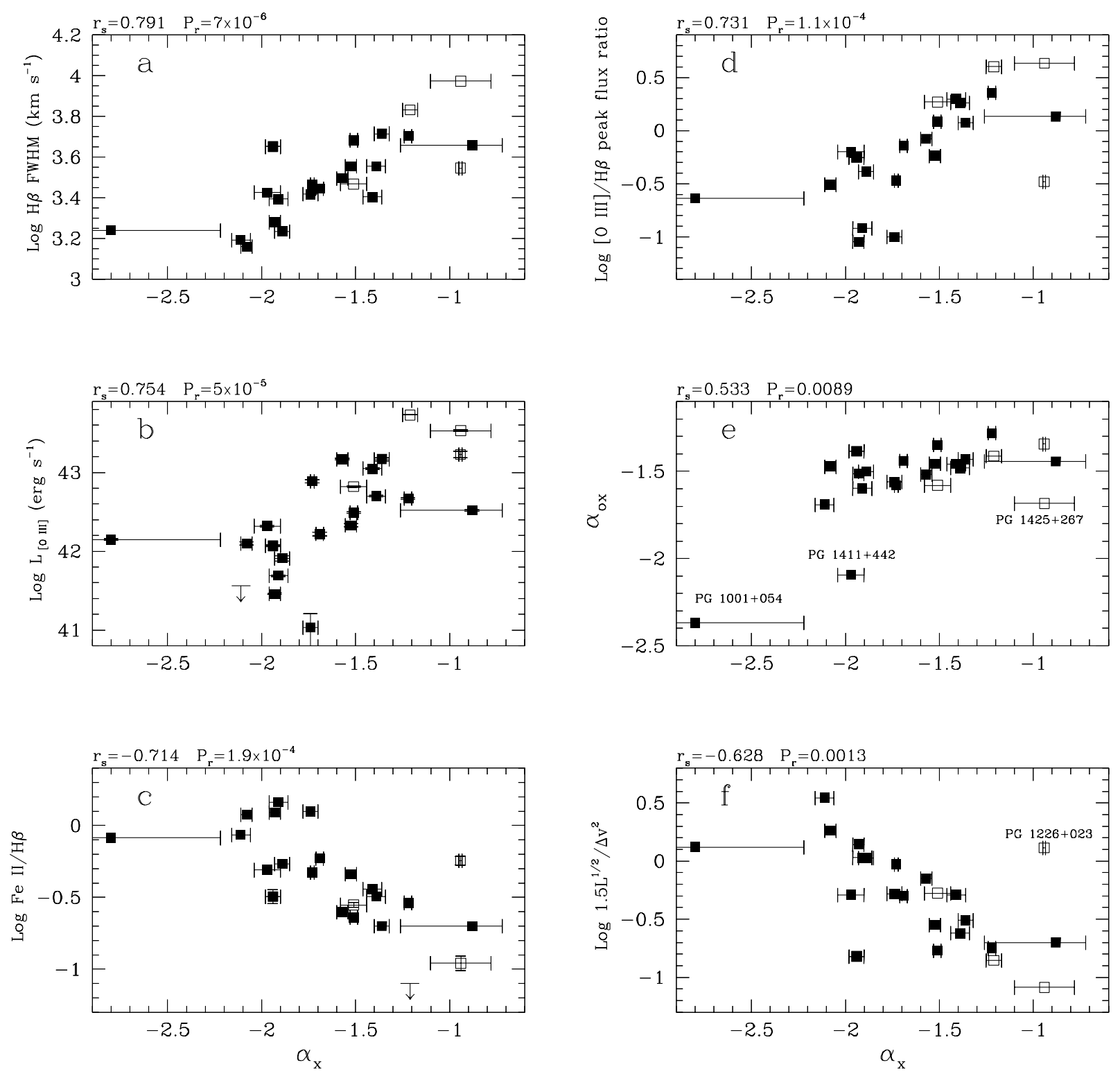

FIG. 5.-Various correlations with $\alpha_{x}$. (a-d) Emission-line parameters that correlate strongly with $\alpha_{x}$. Open symbols are for radio-loud quasars. The $\alpha_{x}$ versus $\mathrm{H} \beta$ FWHM correlation is the strongest in our sample. Note that the X-ray-weak quasars do not stand out in these correlations; they thus have the "right" spectral slope but the "wrong" luminosity. $(e)$ Note the trend of increasing $\alpha_{o x}$ with increasing $\alpha_{x}$, which indicates that a steep $\alpha_{x}$ tends to be associated with a weak $2 \mathrm{keV}$ flux rather than a strong $0.3 \mathrm{keV}$ flux. The position of the X-ray-weak quasars are marked. $(f)$ The $Y$-axis is a measure of $L / L_{\mathrm{Edd}}$ under some simplified assumptions, as described in $\S 4.7$.

tribution for RLQs and RQQs is displayed in Figure 6. To construct it, we used the mean $L_{14.25}$ (Table 5), the mean $\alpha_{o}$, the mean $\alpha_{x}$, and the mean $\alpha_{o x}$ in our sample. We excluded from the mean the three X-ray-weak quasars, and PG $1114+445$, where $\alpha_{x}$ is highly uncertain because of the presence of a warm absorber. The mean spectra were extended above $2 \mathrm{keV}$ assuming a slope of -1 for RQQs and -0.7 for RLQs. The Mathews \& Ferland (1987, hereafter MF) quasar energy distribution is also displayed for the purpose of comparison. The MF shape assumes a steep soft component with a break to the hard X-ray slope above $0.3 \mathrm{keV}$, and it therefore significantly underestimates the soft X-ray flux at $\sim 0.2-1 \mathrm{keV}$.

RLQs tend to be somewhat stronger hard X-ray sources than RQQs. This trend, together with the flatter X-ray slope of RLQs, was interpreted by Wilkes \& Elvis (1987) as possible evidence for a two-component model. In this interpre- tation RLQs have the same hard X-ray component with $\alpha_{x} \sim-1$ as in RQQs, with an additional contribution from a flatter $\alpha_{x} \sim-0.5$ component, making their overall X-ray emission flatter and brighter. The additional X-ray component in RLQs could be related to the radio jet, e.g., through inverse Compton scattering. The composite spectrum suggests that although RLQs are brighter at $2 \mathrm{keV}$, they may actually be fainter at lower energy because of their flatter $\alpha_{x}$. The RLQ composite is based only on four objects and is therefore rather uncertain. In addition, the results of Sanders et al. (1989, § III c) suggest that RLQs in the PG sample are about twice as bright at $2 \mathrm{keV}$ compared with RQQs of similar optical luminosity, rather than the $\sim 30 \%$ found for the composite; thus the difference in PSPC $\alpha_{x}$ would imply a smaller difference at $0.2 \mathrm{keV}$ than is shown in the composite. If RLQs are indeed weaker than RQQs at $0.2 \mathrm{keV}$, then the two-component model suggested above 


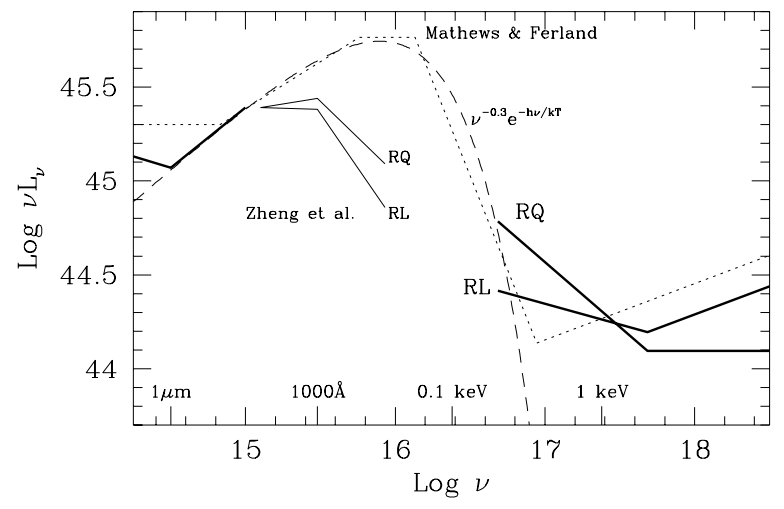

FIG. 6.-Composite optical-soft X-ray spectrum for the RQQs and RLQs in our sample (thick solid line). The three X-ray-weak quasars, and PG $1114+445$, which is affected by a warm absorber, were excluded from the composite. The composite uses $\left\langle\alpha_{o x}\right\rangle=1.482( \pm 0.025)$ and $\left\langle\alpha_{x}\right\rangle=$ $=1.69( \pm 0.07)$ for the RQQs and $\left\langle\alpha_{o x}\right\rangle=1.445( \pm 0.07)$ and $\left\langle\alpha_{x}\right\rangle=$ $1.22( \pm 0.16)$ for the RLQs. Note that despite the fact that RLQs are brighter at $2 \mathrm{keV}$, they are fainter at $0.3 \mathrm{keV}$. The Mathews \& Ferland (1987) spectral shape assumes a hard X-ray power law down to $0.3 \mathrm{keV}$ and a very steep component below $0.3 \mathrm{keV}$. This spectral shape is inconsistent with the PSPC results. The far-UV emission can be parameterized as a cutoff power law. The spectrum shown here has $T_{\text {cut }}=5 \times 10^{5} \mathrm{~K}$, which is close to the upper limit on $T_{\text {cut }}$. The Zheng et al. (1996) FUV composites for RLQs and RQQs are plotted in a thin solid line. They suggest that the FUV power law extends into the soft X-ray regime, with no extreme UV spectral break and no steep soft component below $0.2 \mathrm{keV}$.

would not be valid, and RLQs need to have a different $\mathrm{X}$-ray emission process, rather than just an additional component.

The difference between RLQs and RQQs may actually be unrelated to the radio emission properties, as discussed in $\S 4.4$.

Figure 6 also displays a simple cutoff power-law model of the form $L_{v} \propto v^{\alpha_{o}} e^{-h v / k T_{\text {cut }}}$ with $\alpha_{o}=-0.3$ and $T_{\text {cut }}=5.4$ $\times 10^{5} \mathrm{~K}$. This is an alternative way to interpolate between the UV and soft X-ray emission, and it is also a reasonable approximation for an optically thick thermal component. The lack of a very steep low-energy component down to 0.2 $\mathrm{keV}$ allows us to set an upper limit on $T_{\text {cut }}$. The upper limit is set using $\alpha_{o}$ and $\alpha_{o s}$, the slope from $3000 \AA$ to rest-frame $0.15(1+z) \mathrm{keV}$ (the lowest energy where the Galactic absorption correction error is $\leq 30 \%$ ), given by

$$
\alpha_{o s^{\prime}}=\left[2.685 \alpha_{o x}-\left(17.685-\log v_{s^{\prime}}\right) \alpha_{x}\right] / \log \left(v_{s^{\prime}} / 10^{15}\right),
$$

where $\log v_{s^{\prime}}=16.560+\log (1+z)$.The upper limit on the cutoff temperature $T_{\text {cut }}^{\mathrm{ul}}$ is related to the spectral slopes by

$$
T_{\text {cut }}^{\mathrm{ul}}=\frac{4.8 \times 10^{-11}\left(v_{s^{\prime}}-10^{15}\right) \log e}{\left(\alpha_{o}-\alpha_{o s^{\prime}}\right) \log \left(v_{s^{\prime}} / 10^{15}\right)} \mathrm{K} .
$$

We find a rather small dispersion in $T_{\text {cut }}^{\mathrm{ul}}$ with $\left\langle T_{\text {cut }}^{\mathrm{ul}}\right\rangle=$ $(5.5 \pm 2.6) \times 10^{5} \mathrm{~K}$, averaged over the complete sample (Table 4), which corresponds to a cutoff energy of $47 \mathrm{eV}$, or about 3.5 rydbergs. This value of $T_{\text {cut }}^{\mathrm{ul}}$ corresponds very closely to the far-UV continuum shape assumed by MF (see Fig. 6).

Walter et al. (1994) fitted such a cutoff model directly to six quasars and Seyfert galaxies, finding $\left\langle E_{\text {cut }}\right\rangle=63 \pm 12$ $\mathrm{eV}$, or $\left\langle T_{\text {cut }}\right\rangle=(7.3 \pm 1.4) \times 10^{5} \mathrm{~K}$, while Rachen et al. (1996) find, using such a model, $\left\langle T_{\text {cut }}\right\rangle=(6.3 \pm 2.3) \times 10^{5}$ $\mathrm{K}$ for seven quasars and Seyfert galaxies. These values are consistent with our results. The small dispersion in $T_{\text {cut }}$ reflects the small dispersion in $\alpha_{o s}$ in our sample, which is in marked contradiction to the dispersion predicted by thin accretion disk models, as further discussed in $\S 4.5$.

\subsubsection{The Far-UV Continuum}

Zheng et al. (1996) have constructed a composite quasar spectrum based on $H S T$ spectra of 101 quasars at $z>0.33$. They find a far-UV (FUV) slope (1050-350 $\AA$ ) of $\left\langle\alpha_{\mathrm{FUV}}\right\rangle=$ $-1.77 \pm 0.03$ for RQQs and $\left\langle\alpha_{\mathrm{FUV}}\right\rangle=-2.16 \pm 0.03$ for RLQs, with slopes of $\sim-1$ in the 2000-1050 $\AA$ regime. The Zheng et al. mean spectra, presented in Figure 6, together with the PSPC mean spectra, suggest that the FUV powerlaw continuum extends to the soft X-ray band. In the case of RQQs there is remarkable agreement in both slope and normalization of the soft X-ray and the FUV power-law continua. RLQs are predicted to be weaker than RQQs at $\sim 100 \mathrm{eV}$ by both the FUV and the PSPC composites. It thus appears that there is no extreme UV sharp cutoff in quasars, and that the fraction of bolometric luminosity in the FUV regime is significantly smaller than assumed.

The UV to X-ray continuum suggested in Figure 6 is very different from the one predicted by thin accretion disk models ( $\$ 4.5)$ and suggested by photoionization models. In particular, it implies about a 4 times weaker FUV ionizing continuum compared with the MF continuum that was deduced based on the He II $\lambda 1640$ recombination line.

One should note, however, that the Zheng et al. sample is practically disjoint from our low- $z$ sample, so it may still be possible that low- $z$ quasars have a different FUV continuum.

\subsection{Intrinsic Absorption}

As shown in Figure 2, the $\mathrm{H}$ I column deduced from our accurate $21 \mathrm{~cm}$ measurements is consistent for all objects with the best-fit X-ray column. It is quite remarkable that even in our highest $\mathrm{S} / \mathrm{N}$ spectra the $21 \mathrm{~cm}$ and X-ray columns agree to a level of about $1 \times 10^{19} \mathrm{~cm}^{-2}$, or $5 \%-7 \%$. This agreement is remarkable, since the $21 \mathrm{~cm}$ line and the PSPC are actually measuring the columns of different elements. Most of the soft X-ray absorption is due to $\mathrm{He} \mathrm{I}$ or $\mathrm{He}$ II rather than $\mathrm{H} \mathrm{I}$, and the $\mathrm{H} \mathrm{I}$ column is indirectly inferred assuming the column ratio $\mathrm{H} \mathrm{I} / \mathrm{He} \mathrm{I}=10$. The fact that the $21 \mathrm{~cm}$ line and the PSPC give the same $\mathrm{H} \mathrm{I}$ column implies that the $\mathrm{H} \mathrm{I} / \mathrm{He}$ column ratio at high Galactic latitudes must indeed be close to 10 . The dispersion in the $\mathrm{H} \mathrm{I} / \mathrm{He}$ column ratio is lower than $20 \%$ (based on typical quasars in our sample), and may even be lower than $5 \%$ (based on our highest $\mathrm{S} / \mathrm{N}$ spectra). There is therefore no appreciable Galactic column at high Galactic latitudes where the ionized fraction of $\mathrm{H}$ differs significantly from the ionized fraction of $\mathrm{He}$, as found for example in $\mathrm{H}$ II regions (e.g., Osterbrock 1989).

The consistency of the $21 \mathrm{~cm}$ and X-ray columns also indicates that the typical column of cold gas intrinsic to the quasars in our sample must be smaller than the X-ray $N_{\mathrm{H}}$ uncertainty, or about $3 \times 10^{19}(1+z)^{3} \mathrm{~cm}^{-2}$. An additional indication for a lack of an intrinsic cold column in quasars comes from the fact that the strong correlations of $\alpha_{x}$ with the emission-line parameters described above $(\S 3.3)$ become weaker when we use $\alpha_{x}$ from the free $N_{\mathrm{H}}$ fit rather than $\alpha_{x}$ from the fit with $N_{\mathrm{HI}}^{\mathrm{Gal}}$. This indicates that $N_{\mathrm{HI}}^{\mathrm{Gal}}$ is closer to the true $N_{\mathrm{H}}$ than the free $N_{\mathrm{H}}$ (see discussion in Paper I). In our highest $\mathrm{S} / \mathrm{N}$ spectra we can set an upper limit of $\sim 2 \times 10^{19} \mathrm{~cm}^{-2}$ on any intrinsic absorption. As discussed 
in Paper I, the lack of intrinsic X-ray column for most quasars is consistent with more stringent upper limits set by the lack of a Lyman limit edge, as well as the $\mathrm{He} \mathrm{I}$ and the $\mathrm{He}$ II bound-free edges in a few very high $z$ quasars.

Puchnarewicz et al. (1996) suggest that the strong $\alpha_{x}$ versus $\alpha_{o x}$ correlation in their X-ray-selected sample is due to absorption of the optical and soft X-ray emission by cold gas and dust. They show that the $\alpha_{x}$ versus $\alpha_{o x}$ correlation for the 10 objects in Paper I can be explained by a universal spectral shape absorbed by a gas with a column of up to $N_{\mathrm{H}}=3 \times 10^{20} \mathrm{~cm}^{-2}$ (see their Fig. 16). As described above, such absorbing columns are clearly ruled out by our high $\mathrm{S} / \mathrm{N}$ spectra.

The mean $\alpha_{o}$ in the Puchnarewicz et al. (1996) sample is $-0.92 \pm 0.07$, which is significantly steeper than the mean $\alpha_{o}$ for optically selected quasars, e.g., a median of -0.2 for 105 PG quasars (Neugebauer et al. 1987), a median of -0.32 for 718 LBQS quasars (Francis et al. 1991), and $\left\langle\alpha_{o}\right\rangle=-0.36 \pm 0.05$ in our sample. Puchnarewicz et al. suggested that the much flatter $\alpha_{o}$ of the PG quasars is a selection bias, since these quasars were selected by the strength of their UV excess. However, the PG sample was selected on the basis of the color criterion $U-B<-0.44$, which, using the flux transformations of Allen (1973), corresponds to $\alpha_{o} \geq-1.8$. Thus, most of the red quasars discovered by Puchnarewicz et al. (1996) fit into the PG color criterion. The difference between the soft X-ray-selected and optically selected quasars must reflect the true tendency of quasars selected above $0.5 \mathrm{keV}$ to be significantly redder than optically selected quasars. These red quasars may very well be affected by a large absorbing column $\left(N_{\mathrm{H}}>10^{21}\right.$ $\mathrm{cm}^{-2}$ ), as suggested by Puchnarewicz et al.

Intrinsic absorption is common in Seyfert 1 galaxies. About half of the primarily X-ray-selected Seyfert galaxies observed by Turner \& Pounds (1989) using the EXOSAT LE + ME detectors, by Turner, George, \& Mushotzky (1993a) using the ROSAT PSPC, and by Nandra \& Pounds (1994) using the Ginga LAC for a largely overlapping sample, show low-energy absorption, or spectral features inconsistent with the simple power law typically observed above $2 \mathrm{keV}$. Quasars are very different. Excess absorption produces significant spectral features only in one object (PG $1114+445$; see Paper I), i.e., 5\% (1/23) of the objects, and the absorbing gas is partially ionized ("warm") rather than neutral. Given the typical $\mathrm{S} / \mathrm{N}$ in our sample, we estimate that a partially ionized absorber that produces $\tau>0.3$ can be ruled out in most of our objects. We cannot rule out partial absorption, or complete absorption and scattering, by a very high column density $\left(N_{\mathrm{H}}>10^{24} \mathrm{~cm}^{-2}\right)$ gas, since such effects may only suppress the flux level without affecting the spectral shape, and without inducing significant spectral features. As described in $\S 4.8$, we suspect that such strong absorption may indeed be present in $\sim 10 \%(3 / 23)$ of the quasars in our sample (the X-ray-weak quasars).

\subsection{Implications of the Continuum-Continuum Correlations}

The continuum-continuum luminosity correlations found here are all weaker than those found in Paper I. This is mostly due to the three X-ray-weak quasars that were not present in Paper I. For example, in Paper I we found that $f_{0.3 \mathrm{kev}}$ can be predicted to within a factor of 2 , once $f_{1.69 \mu \mathrm{m}}$ is given. This statement is still valid if the four extreme objects labeled in Figure 3 (middle panel) are excluded. The implications of the near-IR versus X-ray luminosity corre- lation on the X-ray variability power spectrum were discussed in Paper I.

In Paper I we noted the similarity $\left\langle\alpha_{o x}\right\rangle=\left\langle\alpha_{x}\right\rangle=$ -1.50 , which was also noted by Brunner et al. (1992) and Turner et al. (1993a). However, we argued there that this similarity is only fortuitous, and that it does not imply that the X-ray power law can be extrapolated into the UV, since the optical slope is significantly different. Here we find that the relation $\left\langle\alpha_{o x}\right\rangle \simeq\left\langle\alpha_{x}\right\rangle$ holds only roughly for the complete sample where $\left\langle\alpha_{o x}\right\rangle=-1.55 \pm 0.24$ and $\left\langle\alpha_{x}\right\rangle=$ $-1.62 \pm 0.45$. This relation does not hold when the sample is broken to the RQQs where $\left\langle\alpha_{o x}\right\rangle=-1.56 \pm 0.26$ and $\left\langle\alpha_{x}\right\rangle=-1.72 \pm 0.41$, and to the RLQs where $\left\langle\alpha_{o x}\right\rangle=$ $-1.51 \pm 0.16$ and $\left\langle\alpha_{x}\right\rangle=-1.15 \pm 0.27$.

A significantly flatter $\left\langle\alpha_{o x}\right\rangle$ is obtained when the three X-ray-weak quasars and the absorbed quasar PG $1114+445$ are excluded. Thus, "normal" RQQs in our sample have $\left\langle\alpha_{o x}\right\rangle=-1.48 \pm 0.10,\left\langle\alpha_{x}\right\rangle=-1.69 \pm 0.27$, while for the RLQs $\left\langle\alpha_{o x}\right\rangle=-1.44 \pm 0.12,\left\langle\alpha_{x}\right\rangle=-1.22$ \pm 0.28 , where the \pm here and above refers to the dispersion about the mean rather than the error in the mean.

The $\alpha_{x}$ versus $\alpha_{o x}$ correlation found here is weaker than in Paper I, owing to the presence of the X-ray-weak quasars. However, the other 20 quasars appear to follow a trend of increasing $\alpha_{x}$ with increasing $\alpha_{o x}$ (Fig. 5e), indicating, as discussed in Paper I, that a steep $\alpha_{x}$ is generally associated with a weak hard X-ray component (at $2 \mathrm{keV}$ ) rather than a strong soft excess. The only object that clearly violates this trend is PG $1626+554$ (Fig. 3), which has both a steep $\alpha_{x}$ and a strong soft excess. Puchnarewicz, Mason, \& Córdova (1994) and Puchnarewicz et al. (1995a, 1995b ) present PSPC spectra of three AGNs with extremely strong soft excess, where $L_{0.2 \mathrm{keV}}>L_{3000 \AA}$. Our sample suggests that such objects are most likely rare, as can also be inferred from the selection criteria of Puchnarewicz et al. (1995a, 1995b), who selected their three objects from the ROSAT WFC all-sky survey, in which only five AGNs were detected. This selection criterion implies that these AGNs must have a very high far-UV flux.

The soft X-ray-selected quasars in the Puchnarewicz et al. (1996) sample have $\left\langle\alpha_{o x}\right\rangle=-1.14 \pm 0.02$, and none of their quasars is "X-ray weak," i.e., with $\alpha_{o x}<-1.6$. The absence of X-ray-weak quasars in their sample is clearly a selection effect. The small survey area implies that most quasars in their sample are optically rather faint $\left(m_{B} \sim\right.$ 18-19). "Normal" $\alpha_{o x}$ quasars in their sample produce a few hundred PSPC counts, but "X-ray-weak" quasars are below their detection threshold. The abundance of "X-rayloud" quasars (i.e., $\alpha_{o x}>-1$ ) in the Puchnarewicz et al. sample is consistent with their rarity in optically selected samples. For example, quasars with $\alpha_{o x}=-1$ are about 20 times fainter at $3000 \AA$ than quasars with $\alpha_{o x}=-1.5$, for the same $L_{x}$. Since the surface density of quasars increases as $\sim f_{B}^{-2.2}$ (see $\S 2.2 .2 .1$ and Fig. 1 in Hartwick \& Schade 1990), where $f_{B}$ is the $B$-band flux, there are about 700 times more of these fainter quasars per $B$ magnitude per square degree. Thus, even if only $0.3 \%$ of quasars at a given $f_{B}$ have $\alpha_{o x}=-1$, there would still be twice as many quasars with $\alpha_{o x}=-1$ than $\alpha_{o x}=-1.5$ per square degree in an X-ray flux-limited sample, such as the Puchnarewicz et al. sample.

\subsection{Implications of the Continuum-Line Correlations}

The presence of the strong correlations of $\alpha_{x}$ with the $\mathrm{H} \beta$ FWHM, with $\left.L_{[\mathrm{O}} \mathrm{III}\right]$, and with the $\mathrm{Fe} \mathrm{II} / \mathrm{H} \beta$ ratio described 
in Paper I is verified here. The correlation coefficients for the complete sample are comparable to or somewhat smaller than those found in Paper I, but since the sample is larger, the significance level is now much higher (Fig. 5). We also report here an additional strong correlation of $\alpha_{x}$ with the ratio of [O III] peak flux to the $\mathrm{H} \beta$ peak flux, which is one of the emission-line parameters measured by Boroson $\&$ Green (1992). The $\alpha_{x}$ versus H $\beta$ FWHM correlation is the strongest correlation we find between any of the X-ray continuum emission parameters and any of the emission-line parameters reported by Boroson \& Green (with the addition of line luminosities reported in Table 5).

The $\alpha_{x}$ versus $\mathrm{H} \beta$ FWHM correlation is much stronger than the well-known $\alpha_{x}$ correlation with radio loudness $\left(r_{\mathrm{S}}=0.26, \mathrm{Pr}=0.23\right.$ in our sample, but see Wilkes \& Elvis 1987 and Shastri et al. 1993 for stronger correlations). Thus, the fact that the average $\alpha_{x}$ in RLQs is significantly flatter than in RQQs ( $\$ 4.1)$ may be completely unrelated to the presence of radio emission, it may just reflect the fact that RLQs tend to have broader lines than RQQs (e.g.,Tables 3 and 5 in Boroson \& Green 1992). This appears to be the case in our sample, where the RLQs follow the same $\alpha_{x}$ versus $\mathrm{H} \beta$ FWHM distribution defined by the RQQs (Fig. $5 a$ ). This intriguing suggestion can be clearly tested by comparing $\alpha_{x}$ for RLQs and RQQs of similar $\mathrm{H} \beta$ FWHM.

Boller, Brandt, \& Fink (1996) studied in detail narrowline Seyfert 1 galaxies (NLS1), and they also find an apparently significant trend of increasing $\alpha_{x}$ with increasing $\mathrm{H} \beta$ FWHM. However, the scatter in their sample is significantly larger than that found here. In particular, they find a large range of $\alpha_{x}$ for $\mathrm{H} \beta \mathrm{FWHM}<2000 \mathrm{~km} \mathrm{~s}^{-1}$, where only a few objects are available in our sample. The overall larger scatter in the Boller et al. (1996) data is probably due in part to the generally larger statistical errors in their $\alpha_{x}$ determinations. Large systematic errors may also be induced by the use of $\mathrm{H} \beta \mathrm{FWHM}$ from a variety of sources. The measured $\mathrm{H} \beta$ FWHM can be sensitive to the exact measuring procedure, such as continuum placement, subtraction of $\mathrm{Fe}$ II blends, and subtraction of the narrow component of the line (produced in the narrow-line region), which may not be well resolved in low-resolution spectra. For example, Shastri et al. (1993) and Boroson \& Green (1992) measured the H $\beta$ FWHM independently for 13 overlapping objects, in eight of which their values differ by more than $1000 \mathrm{~km} \mathrm{~s}^{-1}$. Aside from these technical reasons, the increased scatter may represent a real drop in the strength of the correlation when the luminosity decreases from the quasar level studied here to the Seyfert level studied by Boller et al. (1996). One should also note that intrinsic absorption is probably common in Seyfert 1 galaxies (Turner \& Pounds 1989; Turner et al. 1993a), and such an absorption may lead to a large systematic error in $\alpha_{x}$ unless a high $\mathrm{S} / \mathrm{N}$ spectrum is available indicating that features are present.

Wang et al. (1996) analyzed PSPC spectra of 86 AGNs, including 22 of the 23 quasars from our sample. Their sample is more heterogeneous than ours and includes some high- $z$ quasars and a number of AGNs selected by their strong $\mathrm{Fe}$ II emission. The various correlations found by Wang et al. (1996) are typically similar to, or somewhat weaker than, those found here. For example, their (our) values are $r_{\mathrm{S}}=-0.73(-0.79)$ for $\alpha_{x}$ versus $\mathrm{H} \beta$ FWHM and $r_{\mathrm{S}}=0.65(0.714)$ for $\alpha_{x}$ versus $\mathrm{Fe}$ II/ $/ \mathrm{H} \beta$. The somewhat smaller values found by Wang et al. may result from their inclusion of $z>0.4$ quasars, where $\alpha_{x}$ measures a higher energy slope than measured here, and from their use of a free $N_{\mathrm{H}}$ fit (limited from below by $N_{\mathrm{HI}}^{\mathrm{Gal}}$ ), which increases the random error in $\alpha_{x}$ (see Paper I).

We verify the strong correlation between $L_{\mathrm{H} \beta}$ and $L_{2 \mathrm{keV}}$ found in Paper I. The correlations of the other lines with $\mathrm{X}$-ray luminosity are significantly weaker than found in Paper I, and they are only marginally significant. Corbin (1993) found significant correlations of $L_{2} \mathrm{kev}$ with $\mathrm{Fe} \mathrm{II} / \mathrm{H} \beta$ $\left(r_{\mathrm{S}}=-0.474\right)$, and of $L_{2} \mathrm{kev}$ with the $\mathrm{H} \beta$ asymmetry $\left(r_{\mathrm{s}}=\right.$ $-0.471)$. We find that neither correlation is significant in our sample $\left(r_{\mathrm{S}}=-0.288, \operatorname{Pr}=0.19\right.$ and $r_{\mathrm{S}}=-0.106$, $\operatorname{Pr}=0.63)$. Since we can only test for correlation with $r_{\mathrm{S}}>$ 0.64 , we cannot securely exclude the presence of the correlations reported by Corbin.

As discussed in Paper I, the $\alpha_{x}$ versus $L_{[\mathrm{O} \text { III] }}$ correlation can be used to place a limit on the $\alpha_{x}$ variability on timescales shorter than a few years. Given the scatter in this correlation, we estimate that $\alpha_{x}$ should not vary by significantly more than 0.3 on these timescales.

It is hard to interpret the $\alpha_{x}$ versus [O III] to $\mathrm{H} \beta$ peak flux ratio correlation, since the physical meaning of the [O III] to $\mathrm{H} \beta$ peak flux ratio parameter defined by Boroson \& Green (1992) is rather obscure. The [O III] peak flux is related to the width of $[\mathrm{O} \mathrm{III}]$, and the $[\mathrm{O}$ III] to $\mathrm{H} \beta$ peak flux ratio may thus partly reflect the FWHM ratio of these lines. Thus, this correlation may represent a correlation of the [O III] FWHM with $\alpha_{x}$. High spectral resolution measurements of the $[\mathrm{O} \mathrm{III}]$ line profile are required to test this possibility.

\subsection{Inconsistency with Thin Accretion Disk Models}

Figure 7 presents the continuum emission from two thin accretion disk models. The models are for a disk around a rotating black hole, and viscous stress that scales as $\left(P_{\mathrm{gas}} P_{\mathrm{rad}}\right)^{1 / 2}$, where $P_{\mathrm{rad}}$ is the radiation pressure and $P_{\mathrm{gas}}$ is the gas pressure (Laor \& Netzer 1989). Significant soft $\mathrm{X}$-ray emission is obtained for disks with a high accretion rate and a small inclination. However, as discussed by Fiore

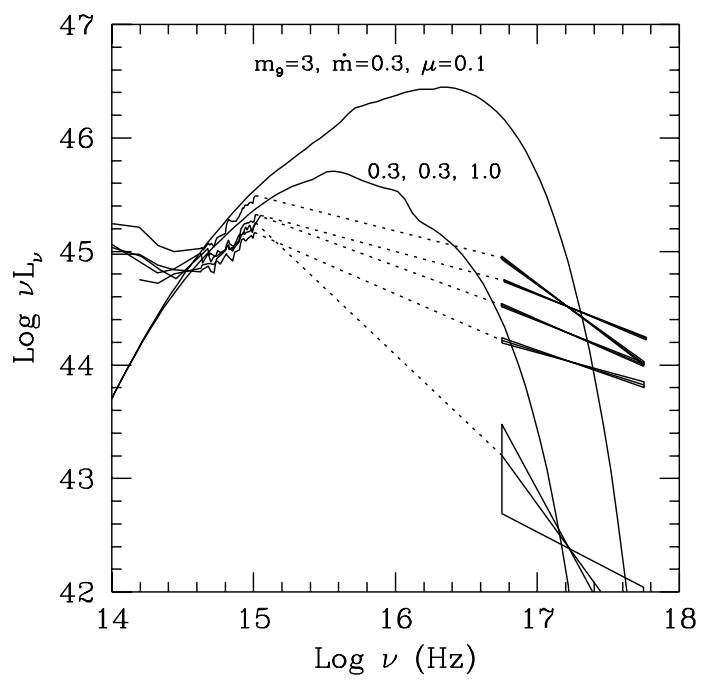

FIG. 7.-Observed energy distribution of some of the quasars in our sample vs. two accretion disk spectra. The disk models are for a rotating black hole, $\alpha=0.1$, and viscous stress $\propto\left(P_{\text {gas }} P_{\text {rad }}\right)^{1 / 2}$ (the other parameters are indicated). Note that although significant soft X-ray flux can be produced by the thin-disk models, the spectral slope is always much steeper than observed. 
et al. (1995), the observed soft X-ray spectral slope is always much flatter than the one produced by a thin "bare" accretion disk model. As noted above, there is no indication in the $0.2-2 \mathrm{keV}$ band for a very steep and soft "accretion disk" component.

Although thin disks cannot reproduce the $0.2-2 \mathrm{keV}$ spectral shape, they may still be able to contribute a significant fraction of the flux at the lowest observed energy, i.e., $0.2-0.3 \mathrm{keV}$, above which a nonthermal power-law component sets in. As noted by Walter et al.(1994) and in Paper $\mathrm{I}$, accretion disk models predict a large dispersion in the optical/soft X-ray flux ratio, and the strong correlation between these fluxes argues against the idea that a thin disk produces both the optical and soft X-ray emission. The arguments put by Walter et al. (1994) and in Paper I were only qualitative, and were not based on actual disk models. Furthermore, the objects in the small sample of Walter et al. were selected from known optically bright AGNs, and they also had to be bright soft X-ray sources, since most spectra were obtained from the ROSAT all-sky survey. Thus, these objects were a priori selected to be bright at both opticalUV and at soft X-rays, and the absence of a large scatter in the UV/soft X-ray flux ratio may just reflect the sample selection criteria. Such selection effects are not present in our sample, since the sample was defined independently of the X-ray properties, and X-ray spectra were obtained for all objects.

Below we describe a detailed calculation of the expected distribution of the optical/soft X-ray flux ratio for a complete optically selected sample based on the thin disk models of Laor \& Netzer (1989), and show that such models cannot be reconciled with the observed distribution of the optical/soft X-ray flux ratio in our complete sample.

The optical/soft X-ray flux ratio, $\alpha_{o s}$, of a given disk model depends on the black hole mass, accretion rate $\dot{m}$, and inclination angle $\theta=\cos ^{-1} \mu$. We now need to determine what distributions of these parameters will be consistent with the observed luminosity function in a complete optically selected sample. The intrinsic distribution of disk inclinations must be random. However, the observed distribution depends on the shape of the luminosity function of quasars, and possible obscuration effects, as described below.

The luminosity function of quasars is parameterized using the number density of quasars per unit volume per unit magnitude, $\Phi \equiv d^{2} N / d M d V$, and it is well fitted by a power law over a restricted range of magnitude $M$. Using Figure 2 in Hartwick \& Schade (1990), we find $\log \Phi=0.55 M+c$ for $z<0.2$ and $\log \Phi=0.66 M+c$ for $0.4<z<0.7$, where $c$ is a constant. Since our sample is restricted to $z<0.4$, we assume $\log \Phi=0.6 M+c$. Using the relation $M=-2.5 \log L+c$, we get $d n / d L \propto L^{-2.5}$, where $n \equiv d N / d V$.

The apparent luminosity of a flat disk, $L_{\mathrm{app}}$, is related to its intrinsic luminosity through $L_{\mathrm{app}}=2 \mu L$, neglecting limb-darkening effects that steepen the $\mu$ dependence and relativistic effects that flatten the $\mu$ dependence. This provides a reasonable approximation in the optical-near-UV regime (see Laor, Netzer, \& Piran 1990). Assuming $d N /$ $d \mu=$ constant, i.e., a uniform distribution of inclination angles for the intrinsic quasar population, we would like to find $d n / d \mu$ for a given $L_{\text {app }}$. When $L_{\text {app }}$ is fixed, $\mu \propto L^{-1}$, and substituting $\mu$ in the expression for $d n / d L$, we get $d n /$ $d \mu \propto \mu^{0.5}$. Thus although the disks are assumed to have a uniform distribution of inclination angles, the observed distribution at a given $L_{\mathrm{app}}$ is biased toward face-on disks.

To reproduce the observed luminosity function, we choose two values of $\dot{m}, 0.1$ and 0.3 , where $\dot{m}$ is measured in units of the Eddington accretion rate. Since $L \propto m_{9} \dot{m}$, where $m_{9}$ is the black hole mass in units of $10^{9} M_{\odot}$, the required mass distribution is $d n / d m_{9} \propto m_{9}^{-2.5}$. The observed number of objects in a flux-limited sample is $d N_{\mathrm{ob}} / d L \propto$ $(d n / d L) V(L)$, where $V(L) \propto L^{3 / 2}$ is the observable volume for a flux-limited sample, such as the BQS sample. We therefore select a mass distribution of $d N_{\mathrm{ob}} / d m_{9} \propto m_{9}^{-1}$.

Figure 8 compares the observed distribution of $\alpha_{o s}$, as a function of $v L_{v}$ at $3000 \AA$, with the one expected from thin accretion disk models with the parameter distribution described above. Thin-disk models cannot account for the very small scatter in $\alpha_{o s}$.

The range of observed disk inclinations may actually be smaller than assumed here. For example, for a certain range of inclinations the disk may be completely obscured by an optically thick torus, as suggested in unification schemes for RQQs (e.g., Antonucci 1993). However, even if $\mu$ is fixed at a given value for all AGNs (say $\mu=1$, which corresponds to the points extending from $\alpha_{o s}=-1.5$ on the left axis to $\log v L_{v}=46.5$ on the bottom axis of Fig. 8), the range in $m_{9}$ and $\dot{m}$ will still produce a range in $\alpha_{o s}$ which is much larger than observed.

The X-ray power-law emission is most likely produced by Comptonization of the thermal disk emission in a hot corona above the disk (e.g., Czerny \& Elvis 1987). The slope and normalization of the power-law component are determined by the temperature and electron scattering optical depth in the corona (e.g., Sunyaev \& Titarchuk 1985; Titarchuk \& Lyubarskij 1995). The small range in $\alpha_{o s}$ implies that some physical mechanism that couples the optical and soft X-ray emission processes must be operating, e.g., through a feedback that regulates both the temperature (see Haardt \& Maraschi 1993) and the optical depth of the corona.

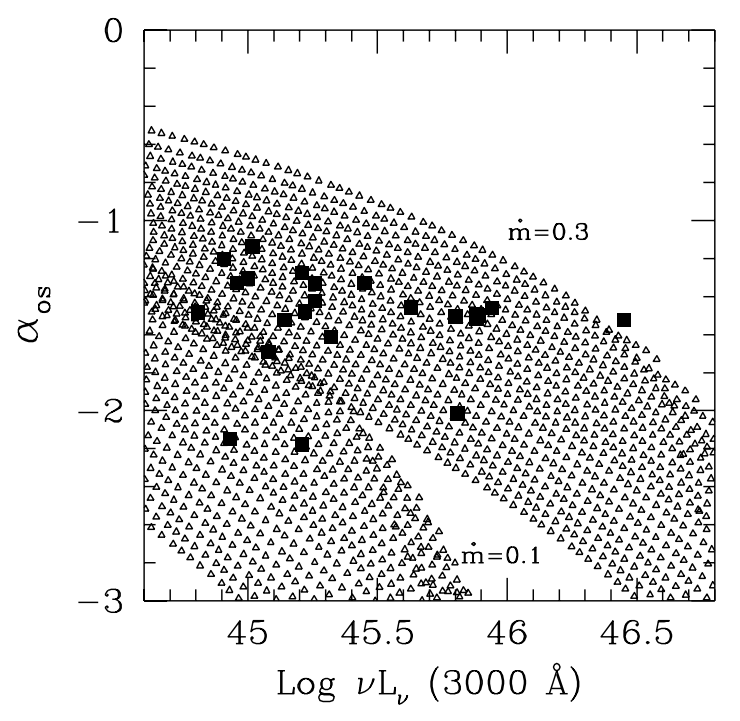

FIG. 8.-Observed $\alpha_{o s}$ vs. luminosity distribution, and the one expected from a distribution of accretion disks that conforms to the observed luminosity function. The observed range in $\alpha_{o s}$ is much smaller than the expected range, indicating that the UV and soft X-ray emission mechanisms are much better correlated than expected from thin-disk models. 
As pointed out by various authors (Ross, Fabian, \& Mineshige 1993; Shimura \& Takahara 1995; Dorrer et al. 1996), and shown in Figure 7, simple thin accretion disks with no corona can produce a significant flux below $1 \mathrm{keV}$. For various disk model parameters $\alpha_{o s}$ can in fact be significantly flatter than observed (Fig. 8), yet such extreme flat optical-soft X-ray spectra are only rarely observed (e.g., Puchnarewicz 1995b). The flattest spectra are expected for disks that are close to edge-on (e.g., Laor et al. 1990), and one therefore needs to assume that such disks are not observable. This is indeed expected in AGN unification schemes that invoke obscuring material close to the disk plane. Alternatively, the accreted material may form a geometrically thick, rather than a thin, configuration close to the center, which would display a smaller inclination dependence.

\subsection{Inconsistency with Optically Thin Free-Free Emission Models}

As was clearly demonstrated by Fiore et al. (1995) for six low-redshift quasars with high S/N PSPC spectra, and by Siemiginowska et al. (1995) using Einstein data for 47 quasars from Elvis et al. (1994), isothermal optically thin pure free-free emission models (Barvainis 1993) cannot fit the observed UV to soft X-ray energy distribution in AGNs. Furthermore, as was pointed out by Kriss (1995) and Hamann et al. (1995), optically thin free-free emission can also be ruled out based on the observed UV line emission.

\subsection{On the Origin of the $\alpha_{x}$ versus $\mathrm{H} \beta F W H M$ Correlation}

What is the physical process behind the $\alpha_{x}$ versus $\mathrm{H} \beta$ FWHM correlation? In Paper I we speculated that this may be either an inclination effect or, possibly, an $L / L_{\text {Edd }}$ effect. Various authors raised the interesting suggestion that steep $\alpha_{x}$ quasars may be analogous to "high "-state Galactic black hole candidates (e.g., White, Fabian, \& Mushotzky 1984; Fiore \& Elvis 1995; Pounds, Done, \& Osborne 1995), which display a steep slope in the soft and the hard X-ray bands when their brightness increases. The physical interpretation for this effect described by Pounds et al. (1995) is that the hard X-ray power law is produced by Comptonization in a hot corona and that, as the object becomes brighter in the optical-UV, Compton cooling of the corona increases, and the corona becomes colder, thus producing a steeper X-ray power law. This is obviously far from being a predictive model, since the coronal heating mechanism is not specified, and it is implicitly assumed that the coronal heating does not increase much as the quasar becomes brighter. However, the narrow $\mathrm{H} \beta$ line profiles provide independent evidence that steep $\alpha_{x}$ quasars may indeed have a higher $L / L_{\mathrm{Edd}}$, as further described below.

The $L / L_{\mathrm{Edd}}$ of quasars can be estimated under two assumptions: (1) The bulk motion of the gas in the broadline region is virialized, i.e., $\Delta v \simeq(G M / r)^{1 / 2}$, where $\Delta v=\mathrm{H} \beta$ FWHM. This gives

$$
\Delta v_{3000}=2.19 m_{9}^{1 / 2} R_{0.1}^{-1 / 2},
$$

where $\Delta v_{3000}=\Delta v / 3000 \mathrm{~km} \mathrm{~s}^{-1}, m_{9}=M / 10^{9} M_{\odot}$, and $R_{0.1}=R / 0.1$ pc. (2) The size of the broad-line region is determined uniquely by the luminosity, $R_{0.1}=L_{46}^{1 / 2} \mathrm{pc}$, where $L_{46}=L_{\text {bolometric }} / 10^{46}$. This scaling is consistent with reverberation line mapping of AGNs (Peterson 1993; Maoz 1995 ) and is theoretically expected if the gas in quasars is dusty (Laor \& Draine 1993; Netzer \& Laor 1994). Combin- ing assumptions 1 and 2 gives

$$
\Delta v_{3000}=2.19 m_{9}^{1 / 2} L_{46}^{-1 / 4},
$$

and thus the mass of the central black hole is

$$
\begin{gathered}
m_{9}=0.21 \Delta v_{3000}^{2} L_{46}^{1 / 2} . \\
\text { Using } L_{\mathrm{Edd}, 46}=12.5 m_{9}, \text { one gets } \\
L / L_{\mathrm{Edd}}=0.38 \Delta v_{3000}^{-2} L_{46}^{1 / 2} .
\end{gathered}
$$

Thus, given the two assumptions made above, narrow-line quasars should indeed have a high $L / L_{\mathrm{Edd}}$, as previously suggested based only on their steep $\alpha_{x}$ and in analogy to Galactic black hole candidates.

To test whether $L / L_{\mathrm{Edd}}$ is indeed the underlying parameter that determines $\alpha_{x}$, rather than just the $\mathrm{H} \beta$ FWHM, we looked at the correlation of $\alpha_{x}$ versus $\Delta v_{3000}^{-2} L_{46}^{1 / 2}$ displayed in Figure $5 f$, where we used the $1.7 \mu \mathrm{m}$ luminosity and the relation $L_{\text {bolometric }}=15 L_{14.25}$ (see Fig. 7 in Laor \& Draine 1993). This correlation is not as strong as the $\alpha_{x}$ versus $\mathrm{H} \beta$ FWHM correlation, but it certainly appears suggestive. Note that $L / L_{\text {Edd }}>1$ for some of the objects in the sample. These values are well above the thin accretion disk limit $\left(L / L_{\mathrm{Edd}}=0.3\right.$; Laor \& Netzer 1989) and suggest a thickdisk configuration. However, the assumptions used above to infer $L / L_{\mathrm{Edd}}$ are more qualitative than quantitative, since both the luminosity and the velocity field in the broad-line region may not be isotropic and therefore the presence of $L / L_{\mathrm{Edd}}>1$ cannot be securely deduced.

The Pounds et al. mechanism implies that a steep $\alpha_{x}$ is associated with a weak hard X-ray component, and, as described in $\S 3.2$, this indeed appears to be the trend in our sample. If the Pounds et al. (1995) mechanism is true, then steep $\alpha_{x}$ AGNs should also have a steep hard X-ray power law. We are currently pursuing this line of research using $A S C A$ and $S A X$ observations of our sample.

An additional hint that a steep $\alpha_{x}$ may indeed be associated with a high $L / L_{\mathrm{Edd}}$ comes from the anecdotal evidence described by Brandt, Pounds, \& Fink (1995), Brandt et al. (1994), Grupe et al. (1995), and Forster \& Halpern (1996), where a number of Seyfert galaxies with a steep $\alpha_{x}$ display rapid, large-amplitude soft X-ray variability, which, as Boller et al. (1996) discuss, may imply a low-mass black hole and thus a high $L / L_{\mathrm{Edd}}$. We are currently pursuing a more systematic study of the soft X-ray variability properties of broad-line versus narrow-line quasars using the ROSAT HRI.

The Pounds et al. (1995) suggestion is very appealing, since it allows a physical explanation for the tight correlation of apparently completely unrelated quantities. Although it is not clear a priori that $\alpha_{x}$ must steepen with increasing $L / L_{\mathrm{Edd}}$, it appears that this is indeed what happens in Galactic black hole candidates.

Wang et al. (1996) also suggested that steep $\alpha_{x}$ objects have a high $L / L_{\text {Edd }}$, based on the fact that the fraction of luminosity emitted in the X-ray regime in thin accretion disk models increases with $L / L_{\mathrm{Edd}}$, as discussed above in $\S 4.5$. However, if this were indeed the physical process behind the $\alpha_{x}$ versus $\mathrm{H} \beta$ FWHM correlation, then one would expect high- $L / L_{\text {Edd }}$ objects to have a high soft X-ray to optical flux ratio, while we find no correlation between $\mathrm{H} \beta$ FWHM and $\alpha_{o s}\left(r_{\mathrm{S}}=-0.079\right.$; Table 5).

We note in passing that one does not need to eliminate the normal broad-line region in narrow-line AGNs, as one of the options suggested by Boller et al. (1996) and Pounds 
et al. (1995). The lines are narrow simply because of the lower black hole mass. The broad-line-emitting gas does not extend much closer to the center in narrow-line AGNs, as it does not extend much closer to the center in other AGNs, simply because of the effects of a higher ionization parameter, and a higher gas density, each of which quenches line emission.

\subsection{The X-Ray-weak Quasars}

Two of the quasars in our sample, the RQQs PG $1001+054$ and PG $1411+442$, and possibly also the RLQ PG $1425+267$, appear to form a distinct group, which we term here "X-ray-weak" quasars, where the normalized $\mathrm{X}$-ray luminosity is a factor of 10-30 smaller than the sample median. The position of these quasars as outliers can be noticed in the near-IR normalized flux distribution (Fig. 3), in the $\alpha_{x}$ versus $\alpha_{o x}$ correlation (Fig. 5e), and in the $\mathrm{H} \beta$ versus $2 \mathrm{keV}$ and $0.3 \mathrm{keV}$ luminosity correlations (Fig. 4). The first two indicators are based on the spectral shape, but the last one is independent of the spectral shape, and it also suggests a deficiency of the X-ray luminosity by a factor of 10-30 relative to the one expected based on the $\mathrm{H} \beta$ luminosity. An apparently bimodal distribution in $\alpha_{o x}$ can also be seen in Figure $5 b$ of Wang et al. (1996), where six of their 86 quasars appear to form a distinct group with $\alpha_{o x}<$ -2 .

No bimodality of $\alpha_{o x}$ is seen in the Avni \& Tananbaum (1986) Einstein study of the PG quasars. All the "X-rayweak" quasars found by the ROS AT PSPC have $\alpha_{o x}<-2$, but none of the quasars detected by Tananbaum et al. (1986) have $\alpha_{o x}<-2$ (see Fig. 8 in Avni \& Tananbaum 1986). The lack of $\alpha_{o x}<-2$ and bimodality in the Tananbaum et al. sample probably reflects its incompleteness, as only $86 \%$ of the quasars they observed were detected.

Although the three X-ray-weak quasars in our sample stand out in luminosity correlations, they conform well to the $\alpha_{x}$ correlations (Figs. $5 a-5 d$ ). They thus have the "right" slope but the "wrong" flux level. Why are these quasars different? A simple answer is that for some unknown reason the X-ray emission mechanism, most likely Comptonization by $T \geq 10^{8} \mathrm{~K}$ electrons, tends to be bimodal, and in about $10 \%$ of quasars (or in all quasars for $\sim 10 \%$ of the time) the $\mathrm{X}$-ray flux level is strongly suppressed, while the spectral slope is not affected. Another option is that these are just normal quasars where the direct X-ray flux happens to be obscured. In this case what we see is only the scattered $\mathrm{X}$-ray flux. Photoionization calculations indicate that a few percent of the direct flux will be scattered, depending on the covering factor of the absorber and the ionization parameter. If the ionization parameter is large enough, then the scattering will be mostly by free electrons, which preserves the spectral shape (see Netzer 1993 and Krolik \& Kriss 1995). Such scattering will explain why the flux level is strongly reduced, while the spectral shape is not affected. Note that the obscuring matter should be transparent in the visible range, as is the case with the absorbing matter in broad absorption line QSOs.

Additional hints toward this interpretation come from the fact that PG $1411+442$ is a broad absorption line quasar (BALQSO; Malkan, Green, \& Hutchings 1987), and the UV absorbing gas may also produce soft X-ray absorption, as may also be the case in PHL 5200 (Mathur, Elvis, \& Singh 1995). In addition, Green \& Mathur (1996) find that BALQSOs observed by $R O S A T$ have $\alpha_{o x} \lesssim-1.8$, i.e., as observed here in the X-ray-weak RQQs. Another hint is provided by the fact that PG $1114+445$ is also somewhat underluminous at $0.3 \mathrm{keV}$ (Fig. $3 b$ ), and this quasar is most likely seen through a warm absorber. The X-ray-weak quasars could therefore be more extreme cases of PG $1114+445$ and have an absorbing column that is large enough to completely absorb the direct soft X-ray emission.

Note that PG $1425+267$ is a RLQ, while all BALQSOs are known to be RQQs (Stocke et al. 1992). It would thus seem implausible to suggest that PG $1425+267$ is a BALQSO. However, PG $1425+267$ has about the same relatively steep $\alpha_{o x}$, compared to other RLQs, as in 3C 351 (Fiore et al. 1993), where X-ray absorption by warm gas is observed together with resonance UV absorption lines (Mathur et al. 1994) which are narrower than in "proper" BALQSOs.

Forthcoming HST spectra of all 23 quasars in our sample will allow us to test whether there is a one-to-one correspondence between X-ray weakness and broad absorption lines, i.e., whether all X-ray-weak quasars are BALQSOs, and not just that all BALQSOs are X-ray weak, as strongly suggested by the Green \& Mathur (1996) and Green et al. (1996) results.

A simple test of whether these are truly " $\mathrm{X}$-ray-weak quasars" or just normal highly absorbed quasars can be done by looking at their hard X-ray emission. If the X-ray column is below $10^{24} \mathrm{~cm}^{-2}$, then the obscuring material would become transparent at $E<10 \mathrm{keV}$, and the observed hard X-ray emission will rise steeply above the cutoff energy, as seen in various highly absorbed AGNs, such as Mrk 3 (Iwasawa et al. 1994), NGC 5506 (Nandra \& Pounds 1994), NGC 6552 (Fukazawa, et al. 1994; Reynolds et al. 1994), and NGC 7582 (Schachter et al. 1996). One may also expect significant spectral features produced by the obscuring material (e.g., Matt et al. 1996), depending on the ionization state of this material. Forthcoming ASCA observations of PG $1411+442$ and PG $1425+267$ will allow us to test this scenario.

Another prediction is that the X-ray-weak quasars should show lower variability compared with other quasars of similar X-ray luminosity. This is true for the following reasons: (1) They are intrinsically more X-ray luminous, and variability amplitude tends to drop with increasing luminosity (Barr \& Mushotzky 1986; also Fig. 9 in Boller et al. 1996). (2) The scattering medium must be significantly larger than the $\mathrm{X}$-ray source, and short-timescale variability will be averaged out. If the X-ray-weak quasars are just due to large-amplitude intrinsic variability of the soft X-ray emission, as seen in some steep-spectrum narrow-line Seyfert 1 galaxies ( $(4.7)$, then one may expect the exact opposite behavior, i.e., these quasars may become significantly brighter at soft X-rays at some stage in the future.

\section{SUMMARY}

We defined a complete sample of 23 optically selected quasars that includes all the PG quasars at $z \leq 0.400$, and $N_{\mathrm{HI}}^{\mathrm{Gal}}<1.9 \times 10^{20} \mathrm{~cm}^{-2}$. Pointed ROSAT PSPC observations were made for all quasars, yielding high $\mathrm{S} / \mathrm{N}$ spectra for most objects. The high quality of the ROSAT spectra allows one to determine the best-fitting $\alpha_{x}$ with about an order of magnitude higher precision compared with previously available $X$-ray spectra. In this paper we report the observations of 13 quasars not described in Paper I, analyze the correlation of the X-ray properties of the complete 
sample with other emission properties, determine the mean $\mathrm{X}$-ray spectra of low- $z$ quasars, and discuss the possible origin of the $\alpha_{x}$ versus $\mathrm{H} \beta$ FWHM correlation, the nature of $\mathrm{X}$-ray-weak quasars, and the physical origin of the soft $\mathrm{X}$-ray emission. Our major results are the following:

1. The spectra of 22 of the 23 quasars are consistent, to within $\sim 10 \%-30 \%$, with a single power-law model over the rest-frame range $0.2-2 \mathrm{keV}$. There is no evidence for significant soft excess emission with respect to the best-fit power law. We place a limit of $\sim 5 \times 10^{19} \mathrm{~cm}^{-2}$ on the amount of excess foreground absorption by cold gas in most of our quasars. The limits are $\sim 1 \times 10^{19} \mathrm{~cm}^{-2}$ in the two highest $\mathrm{S} / \mathrm{N}$ spectra.

2. Significant X-ray absorption by partially ionized gas ("warm absorber") in quasars is rather rare, occurring for $\lesssim 5 \%$ of the population, which is in sharp contrast to lower luminosity AGNs, where significant absorption probably occurs for $\sim 50 \%$ of the population.

3. The average soft X-ray spectral slope for RQQs is $\left\langle\alpha_{x}\right\rangle=-1.72 \pm 0.09$, and it agrees remarkably well with an extrapolation of the mean 1050-350 $\AA$ continuum recently determined by Zheng et al. (1996) for $z>0.33$ quasars. For RLQs $\left\langle\alpha_{x}\right\rangle=-1.15 \pm 0.16$, which suggests that RLQs are weaker than RQQs below $0.2 \mathrm{keV}$, as suggested also by the Zheng et al. mean RLQ continuum. These results suggest that there is no steep soft component below $0.2 \mathrm{keV}$.

4. Extensive correlation analysis of the X-ray continuum emission parameters with optical emission-line parameters indicates that the strongest correlation is between $\alpha_{x}$, and the $\mathrm{H} \beta \mathrm{FWHM}$. A possible explanation for this remarkably strong correlation is a dependence of $\alpha_{x}$ on $L / L_{\mathrm{Edd}}$, as observed in Galactic black hole candidates.

5. There appears to be a distinct class of "X-ray-weak" quasars, which form $\sim 10 \%$ of the population, where the $\mathrm{X}$-ray emission is smaller by a factor of 10-30 than expected based on their luminosity at other bands, and on their $\mathrm{H} \beta$ luminosity.

6 . Thin accretion disk models cannot reproduce the observed $0.2-2 \mathrm{keV}$ spectral shape, and they also cannot reproduce the tight correlation between the optical and soft $\mathrm{X}$-ray emission.
7. The $\mathrm{H} \mathrm{I} / \mathrm{He} \mathrm{I}$ ratio in the ISM at high Galactic latitudes must be within $20 \%$, and possibly within $5 \%$, of the total $\mathrm{H} / \mathrm{He}$ ratio.

The main questions raised by this study are the following:

1. What is the true nature of X-ray-quiet quasars? Are these quasars indeed intrinsically X-ray weak, or are they just highly absorbed but otherwise normal quasars?

2. What physical mechanism is maintaining the strong correlation between the optical-UV and the soft X-ray continuum emission, or, equivalently, maintaining a very small dispersion in the maximum possible far-UV cutoff temperature?

3. What is the physical origin for the strong correlations between $\alpha_{x}$, and $L_{[\mathrm{O} \text { III] }]}, \mathrm{Fe}$ II $/ \mathrm{H} \beta$, and the peak [O III]-to-H $\beta$ flux ratio?

4. Is the soft X-ray emission indeed related to the presence of radio emission, or is it just a spurious relation, and the primary effect is related to the $\mathrm{H} \beta$ line width? Or, put differently, do RLQs and RQQs of similar H $\beta$ FWHM have similar $\alpha_{x}$ ?

Extensions of the ROSAT PSPC survey described in this paper to the hard X-ray regime with $A S C A$ and $S A X$, and to the UV with $H S T$, and soft X-ray variability monitoring with the ROSAT HRI, which are currently being carried out, may provide answers to some of the questions raised above. These studies will also allow us to (1) test whether steep $\alpha_{x}$ quasars have a steep $2-10 \mathrm{keV}$ slope, as expected based on the Pounds et al. (1995) $L / L_{\mathrm{Edd}}$ interpretations; (2) test whether soft X-ray variability is indeed strongly tied to the $\mathrm{H} \beta \mathrm{FWHM}$, as expected if the $\mathrm{H} \beta \mathrm{FWHM}$ is an indicator of $L / L_{\mathrm{Edd}}$; and (3) explore the relation of the UV line emission properties to the ionizing spectral shape.

We thank Niel Brandt, Hagai Netzer, Bev Wills, and an anonymous referee for many useful comments and suggestions. This work was supported in part by NASA grants NAG 5-2087, NAG 5-1618, NAG 5-2496, NAG 5-30934, NAGW 2201 (LTSA), and NASA contract NAS 8-30751. A. L. acknowledges support by LTSA grant NAGW-2144.

\section{REFERENCES}

Allen, C. W. 1973, Astrophysical Quantities (3d ed.; London: Athlone) Antonucci, R. 1993, ARA\&\&A, 31, 473

Avni, Y., \& Tananbaum, H. 1986, ApJ, 305, 83

Barr, P., \& Mushotzky, R. F. 1986, Nature, 320, 421

Barvainis, R. 1993, ApJ, 412, 513

Bevington, P. R. 1969, Data Reduction and Error Analysis for the Physical Sciences (New York: McGraw-Hill)

Boller, Th., Brandt, W. N., \& Fink, H. 1996, A\&A, 305, 53

Boroson, T. A., \& Green, R. F. 1992, ApJS, 80, 109

Brandt, W. N., Fabian, A. C., Nandra, K., Reynolds, C. S., \& Brinkmann, W. 1994, MNRAS, 271, 958

Brandt, W. N., Pounds, K. A., \& Fink, H. 1995, MNRAS, 273, L47

Brunner, H., Friedrich, P., Zimmermann, H.-U., \& Staubert, R. 1992, in

X-Ray Emission from Active Galactic Nuclei and the Cosmic X-Ray

Background, ed. W. Brinkmann \& J. Trümper (Garching: MPI), 198

Canizares, C. R., \& White, J. L. 1989, ApJ, 339, 27

Ciliegi, P., \& Maccacaro, T. 1996, MNRAS, in press

Comastri, A., Setti, G., Zamorani, G., Elvis, M., Giommi, P., Wilkes, B. J. \& McDowell, J. C. 1992, ApJ, 384, 62

Corbin, M. R. 1993, ApJ, 403, L9

Czerny, B., \& Elvis, M. 1987, ApJ, 321, 305

Dickey, J. M., \& Lockman, F. J. 1990, ARA\&A, 28, 215

Dorrer, T., Riffert, H., Staubert, R., \& Ruder, H. 1996, A\&A, in press

Elvis, M., Lockman, F. J., \& Wilkes, B. J. 1989, AJ, 97, 777

Elvis, M., Wilkes, B. J., McDowell, J. C., Green, R. F., Bechtold, J., Willner, S. P., Polomski, E., \& Cutri, R. 1994, ApJS, 95, 1
Ferland, G. F., Korista, K. T., \& Peterson, B. M. 1990, ApJ, 363, L21

Fiore, F., \& Elvis, M. 1995, in Proc. 30th COSPAR Meeting, High Energy Radiation from Galactic and Extragalactic Black Holes, in press

Fiore, F., Elvis, M., Mathur, S., Wilkes, B. J., \& McDowell, J. C. 1993, ApJ, 415,129

Fiore, F., Elvis, M., McDowell, J. C., Siemiginowska, A., \& Wilkes, B. J. 1994, ApJ, 431, 515

Fiore, F., Elvis, M., Siemiginowska, A., Wilkes, B. J., McDowell, J. C., \& Mathur, S. 1995, ApJ, 449, 74

Forster, K., \& Halpern, J. P. 1996, ApJ, 468, 565

Francis, P. J., Hewett, P. C., Foltz, C. B., Chaffee, F. H., Weymann, R. J., \& Morris, S. 1991, ApJ, 373, 465

Fukazawa, Y., et al. 1994, PASJ, 46, L141

Gondhalekar, P. M., Kellett, B. J., Pounds, K. A., Metthews, L., \& Quenby, J. J. 1994, MNRAS, 268, 973

Green, P. J., \& Mathur, S. 1996, ApJ, 462, 637

Green, P. J., et al. 1996, ApJ, 450, 51

Grupe, D., Beuermann, K., Mannheim, K., Thomas, H.-C., Fink, H. H., \& de Martino, D. 1995, A\&A, 300, L21

Haardt, F., \& Maraschi, L. 1993, ApJ, 413, 507

Hamann, F., Shields, J. C., Ferland, G. J., \& Korista, K. T. 1995, ApJ, 454, 688

Hartwick, F. D. A., \& Schade, D. 1990, ARA\&A, 28, 437

Iwasawa, K., Yaqoob, T., Awaki, H., \& Ogasaka, Y. 1994, PASJ, 46, L167

Kellermann, K. I., Saramek, R., Schmidt, M., Shaffer, D. B., \& Green, R. 1989, AJ, 98, 1195 
Kriss, G. 1995, in STScI Symp. Ser. 8, The Analysis of Emission Lines, ed. R. E. Williams \& M. Livio (Cambridge: Cambridge Univ. Press)

Krolik, J. H., \& Kriss, G. A. 1995, ApJ, 447, 512

Kruper, J. S., Urry, C. M., \& Canizares, C. R. 1990, ApJS, 74, 347

Laor, A. 1990, MNRAS, 246, 369

Laor, A., \& Draine, B. T. 1993, ApJ, 402, 441

Laor, A., \& Netzer, H. 1989, MNRAS, 238, 897

Laor, A., Netzer, H., \& Piran, T. 1990, MNRAS, 242, 560

Laor, A., Fiore, F., Elvis, M., Wilkes, B. J., \& McDowell, J. C. 1994, ApJ, 435, 611 (Paper I)

Lawson, A. J., Turner, M. J. L., Williams, O. R., Stewart, G. C., \& Saxton, R. D. 1992, MNRAS, 259, 743

Lockman, F. J., Jahoda, K., \& McCammon, D. 1986, ApJ, 302, 432

Lockman, F. J., \& Savage, B. D. 1995, ApJS, 97, 1

Malkan, M. A., Green, R. F., \& Hutchings, J. B. 1987, ApJ, 322, 729

Maoz, D. 1995, in Reverberation Mapping of the BLR of AGN, ed. P. M. Gondhalekar, K. Horne, \& B. M. Peterson (San Francisco: ASP), 95

Marshall, H. L., Carone, T. E., Shull, J. M., Malkan, M. A., \& Elvis, M. 1996, ApJ, 457, 169

Masnou, J-L, Wilkes, B. J., Elvis, M., Arnaud, K. A., \& McDowell, J. C. 1992, A\&A, 253, 35

Mathews, W. G., \& Ferland, G. J. 1987, ApJ, 323, 456 (MF)

Mathur, S., Wilkes, B., Elvis, M., \& Fiore, F. 1994, ApJ, 434, 493

Mathur, S., Elvis, M., \& Singh, K. P. 1995, ApJ, 455, L9

Matt, G., et al. 1996, MNRAS, 281, L69

Morrison, R., \& McCammon, D. 1983, ApJ, 270, 119

Murphy, E. M., Lockman, F. J., Laor, A., \& Elvis, M. 1996, ApJS, 105, 369

Mushotzky, R. F. 1984, Adv. Space Res., 3(10), 157

Mushotzky, R. F., Done, C., \& Pounds, K. A. 1993, ARA\&A, 31, 717

Nandra, K., George, I. M., Turner, T. J., \& Fukazawa, Y. 1996, ApJ, 464, 165

Nandra, K., \& Pounds, K. A. 1994, MNRAS, 268, 405

Netzer, H. 1993, ApJ, 411, 594

Netzer, H., \& Laor, A. 1993, ApJ, 404, L51

Neugebauer, G., Green, R. F., Mathews, K., Schmidt, M., Soifer, B. T., \& Bennet, J. 1987, ApJS, 63, 615

Osterbrock, D. E. 1989, Astrophysics of Gaseous Nebulae and Active Galactic Nuclei (Mill Valley: University Science Books)

Peterson, B. 1993, PASP, 105, 247

Pounds, K. A., Done, C., \& Osborne, J. P. 1995, MNRAS, 277, L5

Press, W. H., Flannery, B. P., Teukolsky, S. A., \& Vetterling, W. T. 1989, Numerical Recipes: The Art of Scientific Computing (Cambridge: Cambridge Univ. Press)

Puchnarewicz, E. M., Branduardi-Raymont, G., Mason, K. O., \& Sekiguchi, K. 1995a, MNRAS, 276, 1281
Puchnarewicz, E. M., Mason, K. O., \& Córdova, F. A. 1994, MNRAS, 270, 663

Puchnarewicz, E. M., Mason, K. O., Siemiginowska, A., \& Pounds, K. A. 1995b, MNRAS, 276, 20

Puchnarewicz, E. M., et al. 1996, MNRAS, 281, 1243

Rachen, J. P., Mannheim, K., \& Biermann, P. L. 1996, A\&A, 310, 371

Reynolds, C. S., Fabian, A. C., Makishima, K., Fukazawa, Y., \& Tamura, T. 1994, MNRAS, 268, L55

Ross, R. R., Fabian, A. C., \& Mineshige, S. 1993, MNRAS, 258, 189

Sanders, D. B., Phinney, E. S., Neugebauer, G., Soifer, B. T., \& Mathews, K. 1989, ApJ, 347, 29

Savage, B. D., et al. 1993, ApJ, 413, 116

Saxton, R. D., Turner, M. J. L., Williams, O. R., Stewart, G. C., Ohashi, T., \& Kii, T. 1993, MNRAS, 262, 63

Schachter, J. F., et al. 1996, in preparation

Schartel, N., et al. 1996, ApJ, in press

Schmidt, M., \& Green, R. F. 1983, ApJ, 269, 352

Shastri, P., Wilkes, B. J., Elvis, M., \& McDowell, J. 1993, ApJ, 410, 29

Shimura, T., \& Takahara, F. 1995, ApJ, 440, 610

Siemiginowska, A., Kuhn, O., Elvis, M., Fiore, F., McDowell, J. C., \& Wilkes, B. J. 1995, ApJ, 454, 77

Stocke, J. T., Morris, S. L., Weymann, R. J., \& Foltz, C. B. 1992, ApJ, 396, 487

Sunyaev, R. A., \& Titarchuk, L. 1985, A\&A, 143, 374

Tananbaum, H., Avni, Y., Green, R. F., Schmidt, M., \& Zamorani, G. 1986, ApJ, 305, 57

Titarchuk, L., \& Lyubarskij, Y. 1995, ApJ, 450, 876

Turner, T. J., George, I. M., \& Mushotzky, R. F. 1993a, ApJ, 412, 72

Turner, T. J., Nandra, K., George, I. M., Fabian, A. C., \& Pounds, K. A. 1993b, ApJ, 419, 127

Turner, T. J., \& Pounds, K. A. 1989, MNRAS, 240, 833

Ulrich, M.-H., \& Molendi, S. 1996, ApJ, 457, 77

Véron-Cetty, M. P., \& Véron, P. 1991, A Catalogue of Quasars and Active Nuclei (5th ed.; Munich: ESO)

Walter, R., \& Fink, H. H. 1993, A\&A, 274, 105

Walter, R., Orr, A., Courvoisier, T. J.-L., Fink, H. H., Makino, F., Otani, C., \& Wamsteker, W. 1994, A\&A, 285, 119

Wang, T., Brinkmann, W., \& Bergeron, J. 1996, A\&A, 309, 81

White, N. E., Fabian, A. C., \& Mushotzky, R. F. 1984, A\&A, 133, L9

Wilkes, B. J., \& Elvis, M. 1987, ApJ, 323, 243

Williams, O. R., et al. 1992, ApJ, 389, 157

Yaqoob, T., et al. 1994, PASJ, 46, L49

Zheng, W., Kriss, G. A., Telfer, R. C., Grimes, J. P., \& Davidsen, A. F. 1996, ApJ, in press 